\title{
Research Article \\ Salivary Redox Biomarkers in Insulin Resistance: Preclinical Studies in an Animal Model
}

\author{
Mateusz Maciejczyk (D), ${ }^{1}$ Cezary Pawlukianiec, ${ }^{2}$ Małgorzata Żendzian-Piotrowska, ${ }^{1}$ \\ Jerzy Robert Ladny, ${ }^{3}$ and Anna Zalewska ${ }^{4}{ }^{4}$ \\ ${ }^{1}$ Department of Hygiene, Epidemiology and Ergonomics, Medical University of Bialystok, 2C Adama Mickiewicza Street, \\ 15-022 Bialystok, Poland \\ ${ }^{2}$ Students Scientific Club "Biochemistry of Civilization Diseases" at the Department of Hygiene, Epidemiology and Ergonomics, \\ Medical University of Bialystok, 2c Mickiewicza Street, 15-233 Bialystok, Poland \\ ${ }^{3}$ Department of Emergency Medicine, Medical University of Bialystok, 2C Adama Mickiewicza Street, 15-022 Bialystok, Poland \\ ${ }^{4}$ Department of Restorative Dentistry and Experimental Dentistry Laboratory, Medical University of Bialystok, 24A Marii \\ Sklodowskiej-Curie Street, 15-276 Bialystok, Poland
}

Correspondence should be addressed to Mateusz Maciejczyk; mat.maciejczyk@gmail.com

Received 21 July 2021; Accepted 24 August 2021; Published 14 September 2021

Academic Editor: Claudio Cabello-Verrugio

Copyright (c) 2021 Mateusz Maciejczyk et al. This is an open access article distributed under the Creative Commons Attribution License, which permits unrestricted use, distribution, and reproduction in any medium, provided the original work is properly cited.

\begin{abstract}
Insulin resistance (IR) is a condition of impaired tissue response to insulin. Although there are many methods to diagnose IR, new biomarkers are still being sought for early and noninvasive diagnosis of the disease. Of particular interest in laboratory diagnostics is saliva collected in a stress-free, noninvasive, and straightforward manner. The purpose of the study was to evaluate the diagnostic utility of salivary redox biomarkers in preclinical studies in an animal model. The study was conducted on 20 male Wistar rats divided into two equal groups: a standard diet and a high-fat diet (HFD). In all rats fed the HFD, IR was confirmed by an elevated homeostasis model assessment (HOMA-IR) index. We have shown that IR is responsible for the depletion of the enzymatic ( $\downarrow$ superoxide dismutase) and nonenzymatic ( $\downarrow$ ascorbic acid, $\downarrow$ reduced glutathione (GSH)) antioxidant barrier at both the central (serum/plasma) and salivary gland (saliva) levels. In IR rats, we also demonstrated significantly higher concentrations of protein/lipid oxidation ( $\uparrow$ protein carbonyls, $\uparrow 4$-hydroxynoneal (4-HNE)), glycation ( $\uparrow$ advanced glycation end products), and nitration ( $\uparrow 3$-nitrotyrosine) products in both saliva and blood plasma. Salivary nonenzymatic antioxidants and oxidative stress products generally correlate with their blood levels, while GSH and 4-HNE have the highest correlation coefficient. Salivary GSH and 4-HNE correlate with body weight and BMI and indices of carbohydrate metabolism (glucose, insulin, HOMA-IR) and proinflammatory adipokines (leptin, resistin, TNF- $\alpha$ ). These biomarkers differentiate IR from healthy controls with very high sensitivity (100\%) and specificity (100\%). The high diagnostic utility of salivary GSH and 4-HNE is also confirmed by multivariate regression analysis. Summarizing, saliva can be used to assess the systemic antioxidant status and the intensity of systemic oxidative stress. Salivary GSH and 4-HNE may be potential biomarkers of IR progression. There is a need for human clinical trials to evaluate the diagnostic utility of salivary redox biomarkers in IR conditions.
\end{abstract}

\section{Introduction}

One of the most significant medical problems of the 21st century is the increased incidence of type 2 diabetes mellitus (DM2). Nowadays, more than 422 million people worldwide have diabetes, of which about $30-40 \%$ are still undiagnosed
[1]. Several epidemiological studies have shown that DM2 complications cause disability and reduced quality of life for patients with diabetes $[1,2]$. DM2 not only leads to micro- and macrovascular angiopathies but is also a significant cause of premature mortality in developed countries [3]. A key role in DM2 development has been attributed to 
insulin resistance (IR), that is, decreased sensitivity of target tissues to insulin action [4]. IR enhances vascular endothelial proliferation, inflammation, and atherosclerotic plaque formation, mainly due to inhibition of the 3phosphatidylinositol 3-kinase (PI-3K) and mitogenactivated protein kinase (MAP-kinases) pathways. In addition, IR is inextricably linked to obesity [4, 5]. Increased levels of free fatty acids (FFAs), adipokines, and proinflammatory cytokines inhibit insulin action by affecting the insulin receptor substrate (IRS-1) or impairing translocation of the glucose transporter GLUT4 [4-6]. Nevertheless, the common denominator of metabolic disorders in obesity, IR, and $\mathrm{DM} 2$ is also oxidative stress. Increased formation of reactive oxygen species (ROS) occurs not only under ceramide, diacylglycerol (DAG), and triacylglycerol (TAG) accumulation but also impaired insulin signaling and inflammation [7-9]. Oxidative stress has been shown to increase the expression of stress-activated kinases such as protein kinase $\mathrm{C}$ (PKC) and c-Jun N-terminal kinase (JNK), which blocks phosphorylation of IRS-1 tyrosine residues and thus leads to increased blood glucose levels $[6,9,10]$. Overproduction of ROS also results in the phosphorylation of NF- $\kappa \mathrm{B}$ (nuclear factor kappa-light-chain-enhancer of activated B cells) inhibitor, responsible for activating this transcription factor and inducing inflammation $[6,11]$. It also enhances the synthesis of ceramide and, through positive feedback, increases the production of free radicals [12-14]. Therefore, oxidative stress is considered one of the most important pathological factors in the progression of metabolic diseases $[6,9,10,15,16]$. Therefore, it is not surprising that redox biomarkers have been postulated for the diagnosis of IR [17-22].

A biomarker is an objectively measurable change in a biological material, which may indicate a normal physiological state (clinical diagnosis), a pathological state (monitoring of disease progression), or a response to pharmacological treatment (evaluating the effectiveness of drug therapy) [23]. However, despite the tremendous development of diabetology and endocrinology, the current state of knowledge is still not sufficient to rapidly diagnose and treat the metabolic complications of IR. Therefore, alternative and effective diagnostic/treatment methods are constantly being sought [1]. Recently, saliva is of increasing interest in clinical diagnostics. Its advantages include low cost, high durability, and noninvasive and painless collection, including from children and disabled persons [24-27]. Although the main component of saliva is water, it also contains electrolytes, amino acids, proteins, lipids, hormones, vitamins, antioxidants, and oxidation products of biomolecules [28]. These compounds can pass from the blood to saliva by passive (simple diffusion, ultrafiltration, and facilitated diffusion) and active transport as well as through damaged cell membranes $[29,30]$. Thus, saliva contains most of the compounds present in the plasma, which is the basis for its use in medical diagnostics. Despite many studies on the role of oxidative stress in the pathogenesis of IR $[6,9,10,15,16]$, there are no data on the usefulness of salivary redox biomarkers in the diagnosis of insulin resistance. Therefore, our study is the first to evaluate the clinical utility of salivary antioxidants and oxidative stress products in a preclinical study in an animal model. In insulinresistant rats, the activity/concentration of salivary redox biomarkers was compared with their plasma and serum levels and the classical biomarkers of metabolic disturbances.

\section{Results}

2.1. Animal Characteristics. The study was conducted on 20 male Wistar rats divided into two equal groups: a standard diet and a high-fat diet (HFD). Body weight, BMI, and energy intake were significantly higher in the HFD group than in the control group. Interestingly, the food intake in rats fed HFD was notably lower compared to the normally fed rats. Moreover, plasma glucose, insulin, leptin, and resistin concentrations were significantly higher in the HFD group compared to the control. HOMA-IR index was significantly higher in all HFD-fed animals confirming systemic IR (Table 1).

2.2. Enzymatic Antioxidants. Antioxidants are compounds that form a protective barrier in cells, neutralizing the effects of free radicals. Antioxidant enzymes include catalase (CAT), salivary peroxidase $(\mathrm{Px}) /$ glutathione peroxidase (GPx), glutathione reductase (GR), and superoxide dismutase (SOD).

The analysis of enzymatic antioxidants revealed a statistically significant decrease in the activity of salivary $\mathrm{Px}$ $(\downarrow 36 \%, p=0.0029$, Figure $1(\mathrm{e}))$ and salivary SOD $(\downarrow 39 \%, p$ $\leq 0.0001$, Figure $1(\mathrm{~m}))$, as well as serum CAT $(\downarrow 53 \%, p \leq$ 0.0001 , Figure $1(\mathrm{~b}))$ and serum SOD $(\downarrow 61 \%, p \leq 0.0001$, Figure $1(\mathrm{n})$ ) in the HFD group compared to the control. On the other hand, no notable difference in the activity of salivary CAT (Figure 1(a)), salivary GR (Figure 1(i)), serum GPx (Figure 1(f)), and serum GR (Figure 1(j)) between groups was observed.

Moreover, significantly higher saliva/serum ratio of CAT $(\uparrow 150 \%, p \leq 0.0001$, Figure $1(\mathrm{c}))$ and SOD $(64 \%, p \leq 0.0012$, Figure 1(o)) in HFD rats was observed in comparison to the control group.

What is more, we observed a high positive correlation of SOD activity $(r=0.7501, p=0.0001$, Figure $1(p))$ between serum and saliva in HFD rats. There were no notable correlations of the other investigated enzymatic biomarkers.

2.3. Nonenzymatic Antioxidant. Nonenzymatic antioxidants mainly include small molecular weight antioxidants such as ascorbic acid (AA), reduced glutathione (GSH), and uric acid (UA). Assays evaluating nonenzymatic antioxidant revealed that high-fat diet caused a significant decrease in the level of salivary $(\downarrow 52 \%, p \leq 0.0001$, Figure $2(a))$ and plasma AA ( $\downarrow 19 \%, p=0.0014$, Figure $2(\mathrm{~b}))$. Similarly, GSH concentration in the saliva $(\downarrow 58 \%, p \leq 0.0001$, Figure $2(\mathrm{e}))$ and in the plasma $(\downarrow 61 \%, p \leq 0.0001$, Figure $2(\mathrm{f}))$ in the HFD group was significantly lower than in the control group. What is more, a high-fat diet increased the concentration of UA in the saliva $(\uparrow 46 \%, p=0.001$, Figure 2(i)) and the plasma $(\uparrow 46 \%, p=0.0024$, Figure $2(\mathrm{j}))$ of HFD rats comparing to the control. 
TABLE 1: General characteristics of the control and high-fat diet fed (HFD) rats.

\begin{tabular}{lcc}
\hline Parameter & Control group & HFD group \\
\hline Body weight $(\mathrm{g})$ & $388 \pm 34.16$ & $537 \pm 36.52^{* * * *}$ \\
BMI $\left(\mathrm{g} / \mathrm{cm}^{2}\right)$ & $0.66 \pm 0.024$ & $0.90 \pm 0.016^{* * * *}$ \\
Energy intake (g/rat/week) & $206.4 \pm 22.06$ & $248.6 \pm 38.23^{* *}$ \\
Food intake (g/day) & $18.07 \pm 1.93$ & $11.05 \pm 1.70^{* * * *}$ \\
Plasma glucose (mg/dL) & $90.94 \pm 3.31$ & $146.4 \pm 12.54^{* * * *}$ \\
Plasma insulin $(\mathrm{mU} / \mathrm{mL})$ & $78.65 \pm 7.55$ & $172.30 \pm 12.07^{* * * *}$ \\
HOMA-IR & $1.72 \pm 0.18$ & $16.20 \pm 0.65^{* * * *}$ \\
Plasma leptin $(\mathrm{mU} / \mathrm{mL})$ & $26.84 \pm 2.88$ & $49.40 \pm 6.24^{* * * *}$ \\
Plasma resistin $(\mathrm{mU} / \mathrm{mL})$ & $184 \pm 19.12$ & $394 \pm 39.51^{* * * *}$ \\
Plasma TNF- $\alpha(\mathrm{mU} / \mathrm{mL})$ & $1010 \pm 115.2$ & $3248 \pm 588.5^{* * * *}$ \\
\hline
\end{tabular}

Abbreviations: BMI: body mass index; HFD: high-fat diet; HOMA-IR: homeostatic model assessment of insulin resistance; TNF- $\alpha$ : tumor necrosis factor $\alpha$; ${ }^{* *} p<0.01$ vs. control; ${ }^{* * * *} p<0.0001$ vs. control.

A significantly lower saliva/serum ratio of AA $(\downarrow 39 \%, p$ $=0.0006$, Figure 2(c)) in rats fed a high-fat diet was observed compared to the control group. Interestingly, there were no statistical differences in the saliva/plasma ratio of GSH (Figure 2(g)) and UA (Figure 2(k)).

Moreover, a highly positive correlation of GSH content $(r=0.7993, p \leq 0.0001$, Figure 2(h) $)$ in plasma and saliva of HFD rats was noticed. AA $(r=0.5334, p=0.0154$, Figure $2(\mathrm{~d}))$ and UA $(r=0.4943, p=0.0267$, Figure $2(\mathrm{l}))$ levels were also positively correlated between rats' plasma and saliva.

2.4. Oxidative/Nitrosative Damage. Oxidation (carbonyl groups (PC); 4-hydroxynoneal (4-HNE)), glycation (advanced glycation end products (AGE) and nitration (3-nitrotyrosine (3-NT)) products of proteins and lipids are used to assess the damage caused by ROS and RNS. The oxidative and nitrosative stress markers revealed that high-fat diet increased concentrations of salivary, as well as plasma PC ( $\uparrow 71 \%, p=0.0018$, Figure $3(\mathrm{a}) ; \uparrow 46 \%, p \leq$ 0.0001 , Figure 3(b), respectively), $3-\mathrm{NT}(\uparrow 6 \%, p=0.0235$, Figure 3(e); $\uparrow 31 \%, p=0.0007$, Figure 3(f)), AGE ( $\uparrow 51 \% p$ $\leq 0.0001$, Figure $3(\mathrm{i}) ; \uparrow 92 \%, p \leq 0.0001$, Figure $3(\mathrm{j}))$, and 4-HNE ( $\uparrow 292 \% p \leq 0.0001$, Figure $3(\mathrm{~m}) ; \uparrow 140 \%, p \leq$ 0.0001 , Figure $3(\mathrm{n}))$.

A significantly lower saliva/serum ratio of 3-NT $(\downarrow 21 \%$, $p=0.0182$, Figure $3(\mathrm{~g}))$ in the HFD group compared to the control group was noticed. On the other hand, in HFD rats, a notably higher saliva/serum ratio of 4 - HNE $(\uparrow 66 \%, p=$ 0.0008 , Figure $3(\mathrm{o}))$ was observed compared to the control group.

Interestingly, significant positive correlations of all of the oxidative and nitrosative damage products such as PC, 3NT, AGE, and 4-HNE ( $r=0.4727, p=0.0353$, Figure $3(\mathrm{~d})$; $r=0.4789, p=0.0327$, Figure $3(\mathrm{~h}) ; r=0.4777, p=0.0331$, Figure 3(l); $r=0.8341, p \leq 0.0001$, Figure $3(\mathrm{p})$, respectively) were observed between plasma and saliva in the HFD group.
2.5. Correlations with Metabolic Parameters. The analysis of the metabolic parameters and investigated biomarkers revealed highly positive correlations between salivary CAT and salivary 4 -HNE, as well as plasma glucose HOMA-IR, and BMI $(r=0.693, p=0.026 ; r=0.651, p=0.041 ; r=$ $0.712, p=0.021 ; r=0.715, p=0.02$, respectively). Interestingly, the activity of salivary SOD was correlated only with plasma resistin $(r=-0.851, p=0.002)$. Moreover, the activity of salivary GSH correlated negatively with BW $(r=-0.667, p=0.035)$ and BMI $(r=-0.889, p=0.001)$, as well as plasma glucose $(r=-0.79, p=0.006)$, insulin $(r=-0.783, p=0.007)$, HOMA-IR $(r=-0.936, p=0.00007$ ), and proinflammatory adipokines (leptin: $r=-0.757, p=$ 0.011 ; resistin: $r=0.685, p=0.029$; TNF- $\alpha: r=-0.639, p=$ $0.047)$. What is more, we have shown highly positive correlation between the salivary UA concentration and plasma leptin, plasma glucose, BW, and BMI $(r=0.92, p \leq 0.0001$; $r=0.777, p=0.008 ; r=0.691, p=0.027 ; r=0.754, p=$ 0.012 , respectively). Furthermore, we observed the positive correlations of salivary 4-HNE and resistin $(r=0.776, p=$ $0.008)$. However, salivary 4-HNE correlated also with BW $(r=0.605, p=0.044)$ and BMI $(r=0.854, p=0.002)$, as well as indicators of carbohydrate metabolism-glucose $(r=0.716$, $p=0.02)$ and HOMA-IR $(r=0.592, p=0.05)$ (Figure 4).

2.6. Multiple Regression Analysis. Multiple regression analysis of salivary biomarkers showed that salivary GSH was negatively associated with HOMA-IR $(p=0.0042)$. Moreover, significant association between BMI and the activity of salivary GSH and concentration of salivary 4-HNE were observed. No significant associations between other salivary biomarkers and BMI or HOMA-IR were noticed (Table 2).

2.7. ROC Analysis of the Analyzed Redox Biomarkers. The ROC analysis revealed that most of the salivary redox biomarkers significantly differentiated rats fed a standard diet and a high-fat diet. The assessment of salivary Px, SOD, and GSH (sensitivity $=70 \%$, specificity $=70 \%, p=0.0102$; sensitivity $=90 \%$, specificity $=90 \%, p=0.0004$; sensitivity $=$ $100 \%$, specificity $=100 \%, p=0.0002$ ) clearly distinguished the HFD group from the control. Similarly, the salivary levels of AA, UA, PC, 3-NT, AGE, and 4-HNE (sensitivity $=90 \%$, specificity $=90 \%, p=0.0002$; sensitivity $=80 \%$, specificity $=80 \%, \quad p=0.0025 ; \quad$ sensitivity $=80 \%$, specificity $=80 \%, p=0.0032$; sensitivity $=70 \%$, specificity $=$ $70 \%, p=0.0343$; sensitivity $=90 \%$, specificity $=90 \%, p=$ 0.0009 ; sensitivity $=100 \%$, specificity $=100 \%, p=0.0002$ ) significantly differentiated the HFD rats from the normal diet group. Moreover, number of serum and plasma biomarkers, such as CAT, SOD, GSH, and 4-HNE ( $p=0.0002$ ), were characterized by a $100 \%$ of sensitivity and specificity in differentiating the HFD rats from the control rats (Table 3).

\section{Discussion}

This study is the first to evaluate the clinical utility of salivary redox biomarkers in IR. In preclinical studies in an animal model, we demonstrated that salivary GSH and 4-HNE 


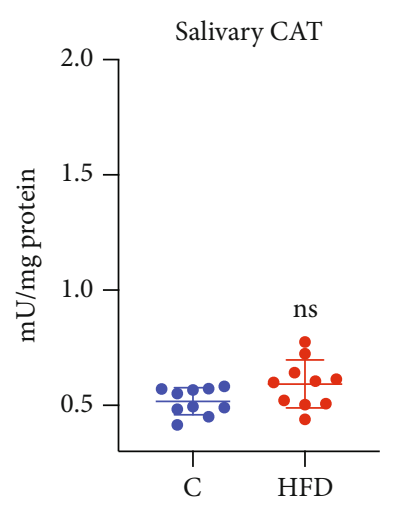

(a)

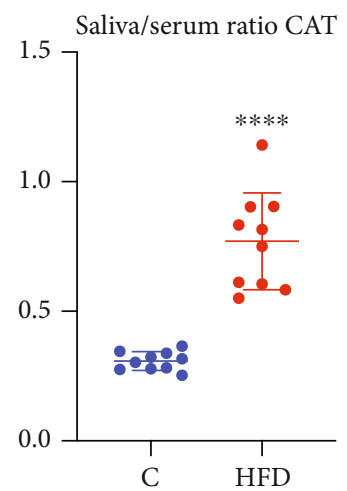

(c)

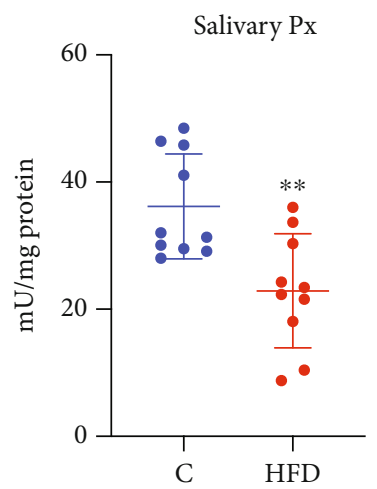

(e)

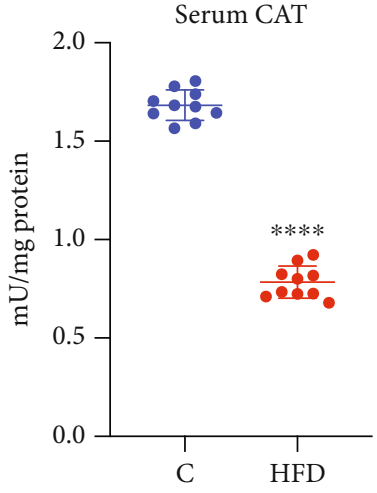

(b)

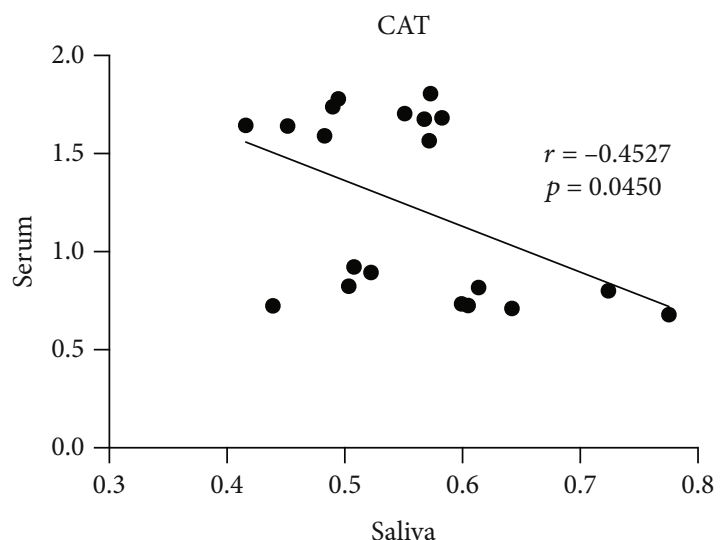

(d)

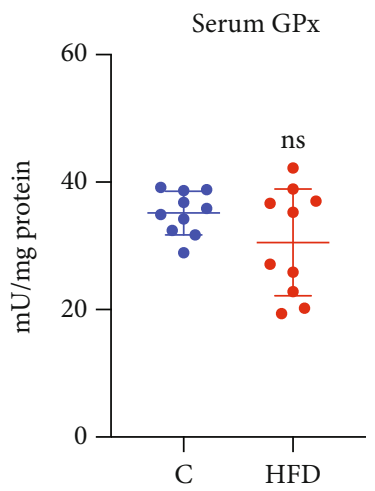

(f)

FIgURE 1: Continued. 


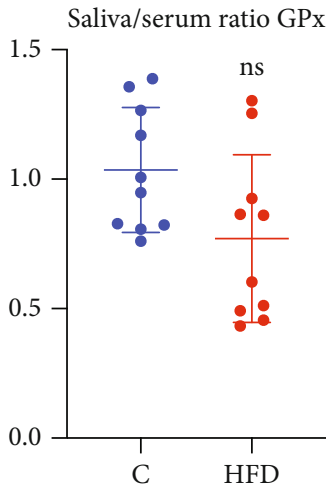

(g)

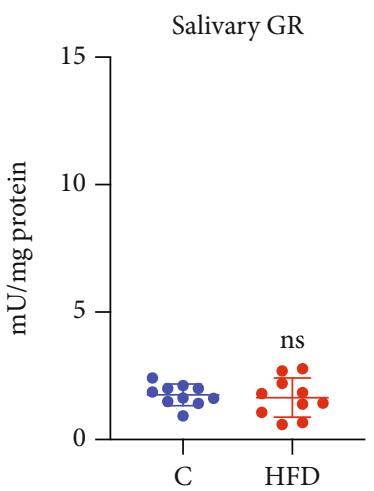

(i)

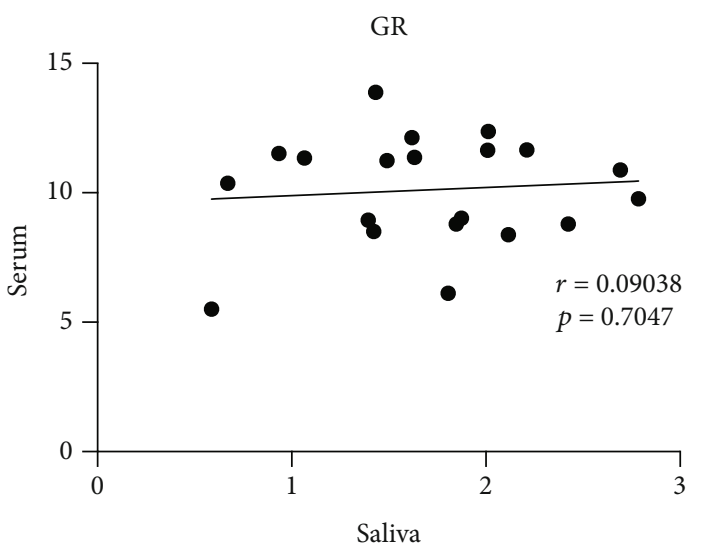

(1)

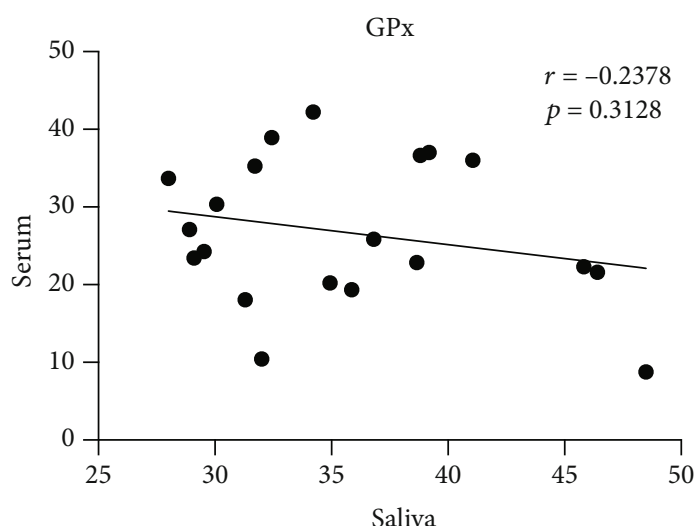

(h)
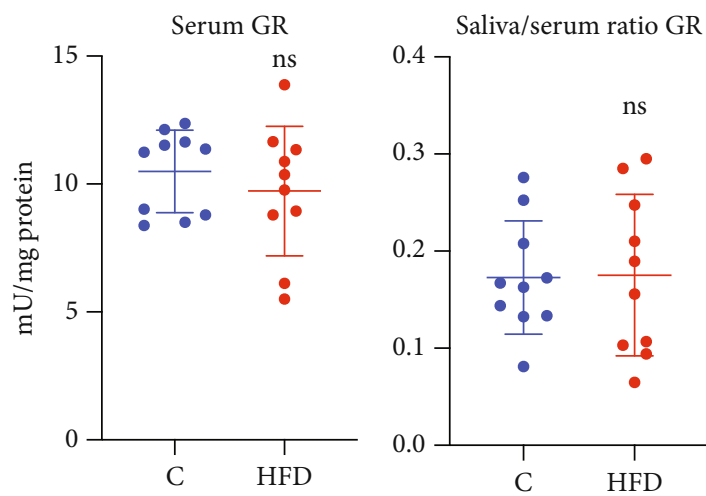

(j)

(k)
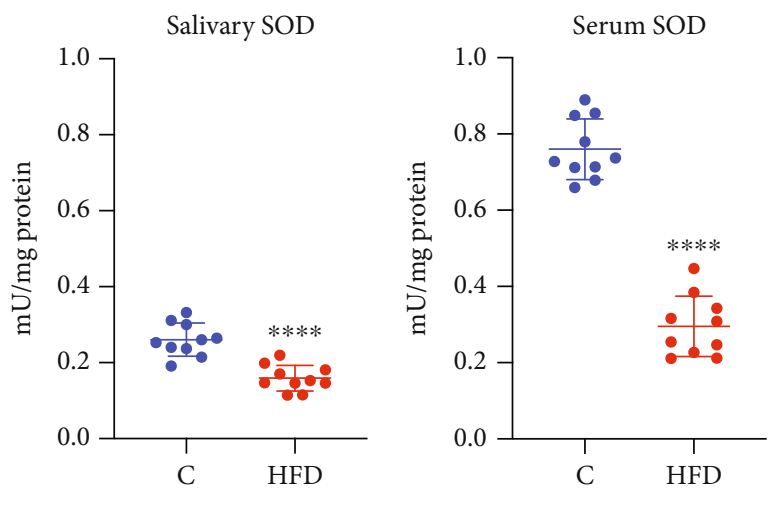

(n)

Figure 1: Continued. 


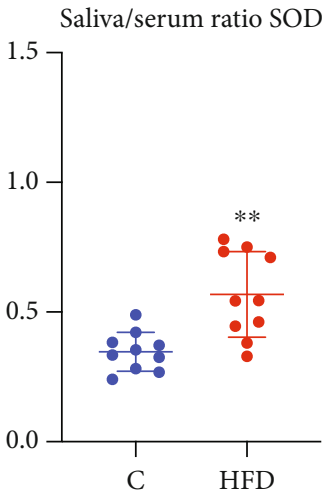

(o)

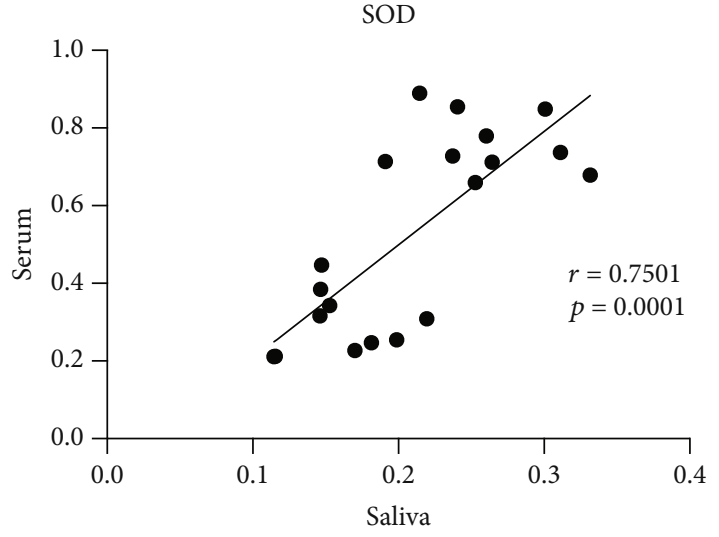

(p)

FIGURE 1: The effect of a high-fat diet (HFD) on salivary and plasma enzymatic antioxidants in rats. Abbreviations: C: control group; HFD: high-fat-diet group; CAT: catalase; GPx: glutathione peroxidase; GR: glutathione reductase; Px: peroxidase; SOD: superoxide dismutase; ${ }^{* *} p<0.01$ vs. control; ${ }^{* * *} p<0.0001$ vs. control; ns: no significance.

levels have high diagnostic value in monitoring IR progression.

IR is a major pathogenetic factor in DM2 preceding the onset of overt hyperglycemia by up to several years [3]. Although decreased tissue sensitivity to insulin is compensated by hyperinsulinemia, IR leads to obesity, hypertension, dyslipidemia, and finally the metabolic syndrome [4]. Therefore, understanding the causes of IR and its treatment is one of the most significant challenges in modern diabetology. The gold standard for IR assessment is the hyperinsulinemic-euglycemic clamp technique [31]. However, this method is not used in routine medical practice due to its high invasiveness and the need to perform the test while the patient is hospitalized. For this reason, insulin resistance is mainly diagnosed using indirect methods. The simplest of them is a measurement of fasting glucose and insulin and calculation of HOMA-IR index (homeostatic model assessment of IR), which is a mathematical model describing interdependence of insulin secretion in response to current basal glycemia [3, 31]. Nevertheless, new biomarkers are still being sought that could noninvasively inform about the progression of IR and its metabolic complications [1]. Of particular diagnostic interest is saliva, which is easy to collect and does not require the specialized equipment or assistance of the medical staff. Saliva can be collected multiple times per day and can replace blood draws in people with clotting disorders, children, or patients with disabilities $[24,25]$. Noninvasive saliva collection reduces patient anxiety, promotes more frequent self-monitoring, and enables the disease diagnosis at an early stage [32]. Unfortunately, the assessment of glucose and insulin in the saliva is not diagnostically relevant because it does not reflect their levels in the blood [33-35]. Nonetheless, there has been considerable recent interest in saliva-based diagnostics focusing on redox biomarkers. Salivary redox biomarkers are commonly used to diagnose hypertension [36, 37], obesity [38-40], chronic kidney disease [41, 42], heart failure $[43,44]$, Hashimoto's disease [45, 46], dementia [47, 48], or cancer $[49,50]$. Considering the critical contribution of oxidative stress in the progression of IR $[6,9,10,15,16]$, we evaluated the salivary antioxidants and products of protein and lipid oxidation/nitration in the saliva of IR rats. We compared the content of salivary redox biomarkers with their plasma levels and the classical indicators of metabolic disorders. We also assessed the diagnostic utility of salivary redox biomarkers using ROC analysis and multivariate regression.

We have shown that IR results in impairment of the salivary/plasma antioxidant barrier, with both enzymatic ( $\downarrow$ SOD,$\downarrow S P x / G P x)$ and nonenzymatic ( $\downarrow$ GSH, $\downarrow$ AA, $\uparrow U A)$ deficiency. Although we did not assess the rate of ROS production, the weakening of antioxidant systems is likely due to increased free radical generation under IR conditions [44]. It is well known that positive energy balance ( $\uparrow$ fat supply) causes increased synthesis of acetyl-CoA and NADP in mitochondria, responsible for ROS overproduction [51, 52]. However, adipose tissue is also an essential source of free radicals in IR [53]. Excess visceral tissue stimulates adipocytes to synthesize chemotactic and adhesive molecules such as MCP1 (monocyte chemoattractant protein 1), VCAM1 (vascular cell adhesion molecule 1), and ICAM (intercellular adhesion molecule 1), which enhance the influx of lymphocytes and macrophages and stimulate the production of proinflammatory cytokines (IL-1, IL-2, TNF- $\alpha$ ) [53-55]. Synthesis of adipokines (resistin and leptin), which mediate inflammation by promoting cytokine efflux, is also increased [53]. Thus, it is not surprising that the antioxidant barrier is diminished, resulting in higher oxidative damage to proteins ( $\uparrow \mathrm{PC}, \uparrow 3-\mathrm{NT}, \uparrow \mathrm{AGE}$ ) and lipids ( $\uparrow 4-\mathrm{HNE})$. Of particular note are disturbances in glutathione metabolism $(\downarrow \mathrm{GSH}$, $\downarrow S P x / G P x)$. GSH participates in hydrogen peroxide degradation and maintains the sulfhydryl groups of proteins in a reduced state [56]. The accumulation of oxidized glutathione (GSSG) in the cell and the formation of protein disulfides with GSH inhibits many enzymes' activity, thereby impairs cell metabolism and energy production [56, 57]. The increased oxidation ( $\uparrow \mathrm{PC}, \uparrow 4-\mathrm{HNE}$ ), glycation ( $\uparrow \mathrm{AGE})$, and nitration ( $\uparrow 3-\mathrm{NT})$ of proteins/lipids observed in our study are also very important. The products of oxidative/nitrosative modifications damage cellular structures and show 


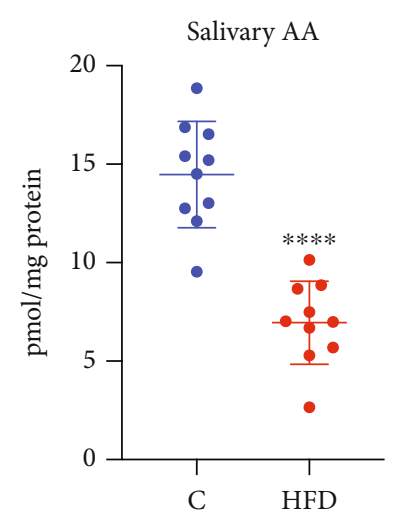

(a)

Saliva/plasma ratio AA

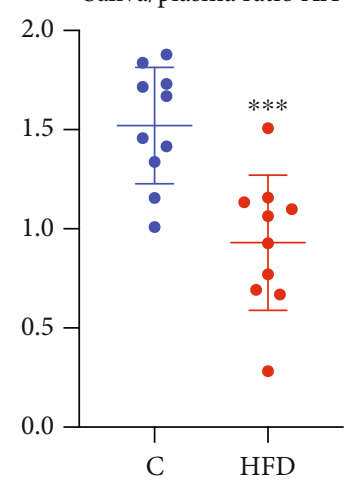

(c)

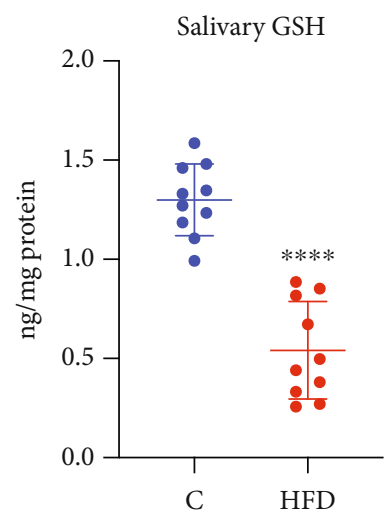

(e)

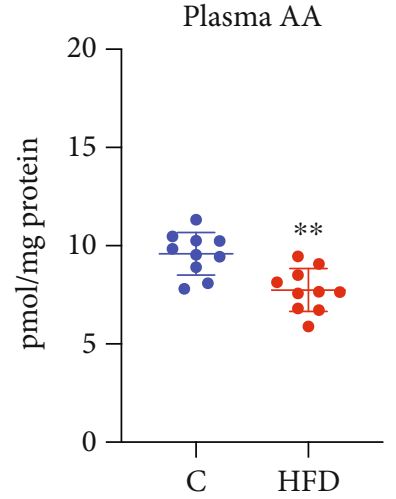

(b)

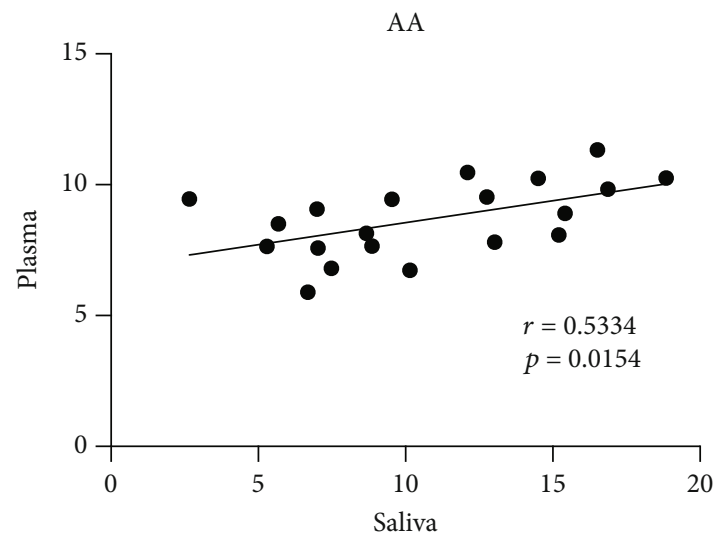

(d)

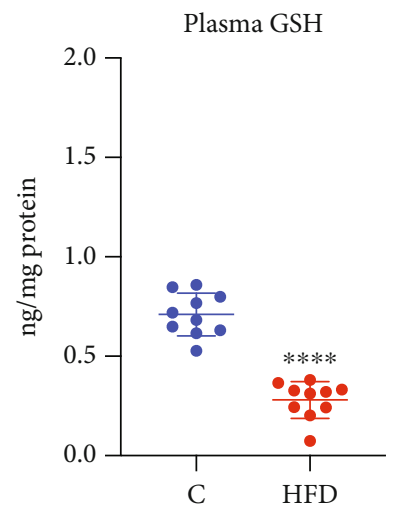

(f)

Figure 2: Continued. 


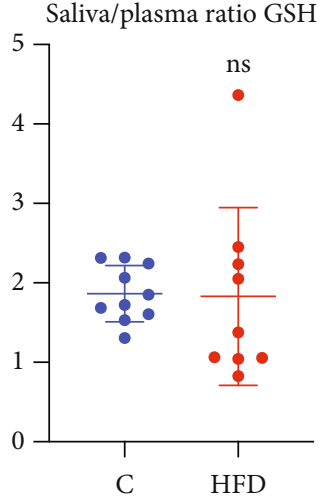

(g)

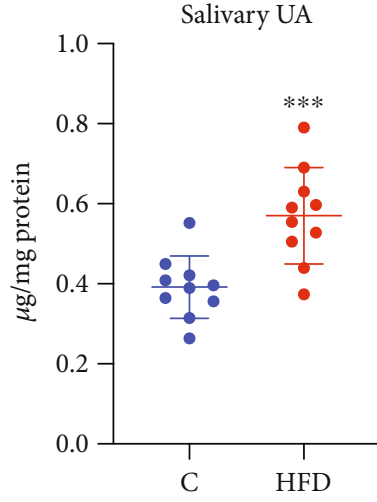

(i)

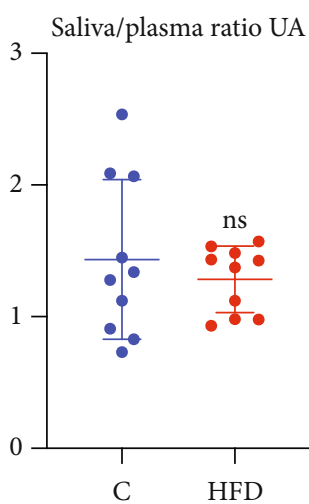

$(\mathrm{k})$

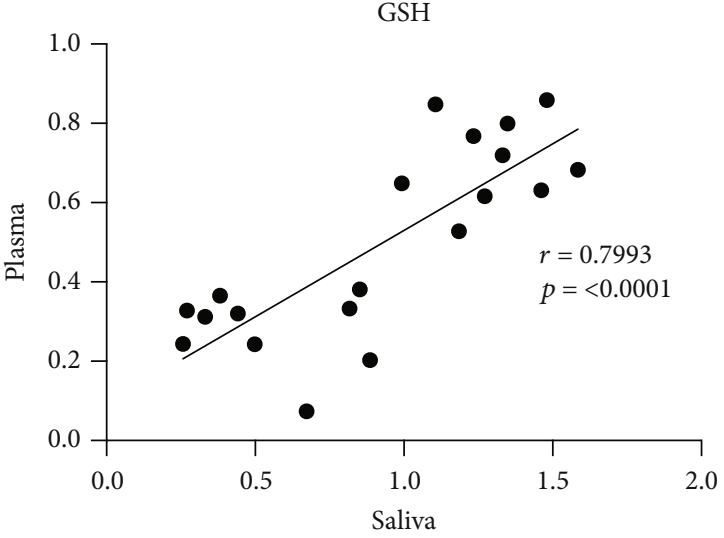

(h)

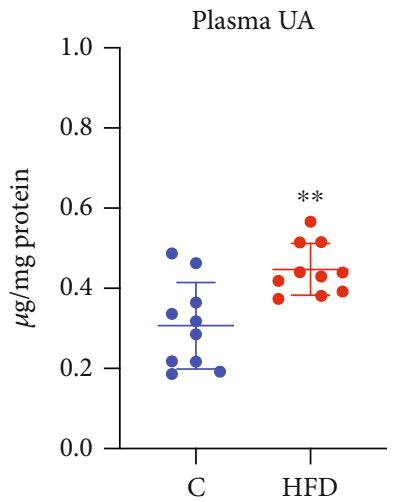

(j)

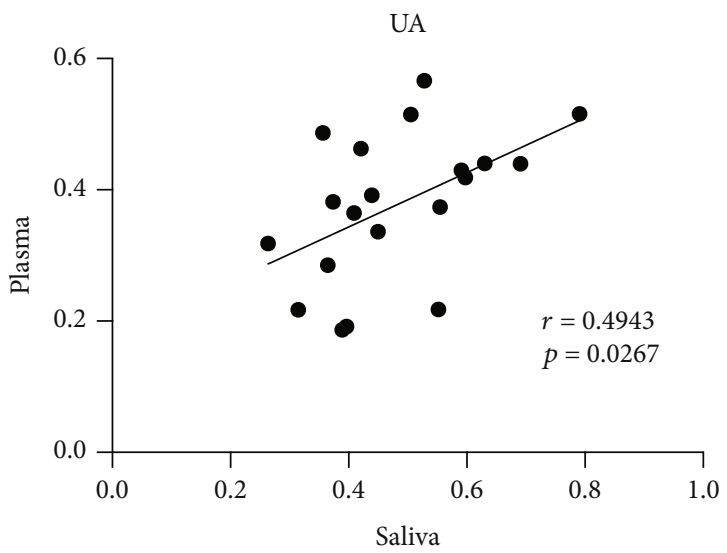

(l)

Figure 2: The effect of a high-fat diet (HFD) on salivary and plasma nonenzymatic antioxidants in rats. Abbreviations: C: control group; HFD: high-fat-diet group; AA: ascorbic acid; GSH: reduced glutathione; UA: uric acid; ${ }^{*} p<0.05$ vs. control; ${ }^{* *} p<0.01$ vs. control; ${ }^{* * *} p$ $<0.001$ vs. control; ${ }^{* * * *} p<0.0001$ vs. control; ns: no significance.

mutagenic and carcinogenic properties. Indeed, compounds such as 4-HNE can form adducts with DNA, promoting instability of the genetic material and several replication errors [58, 59].

A crucial part of our study was to evaluate the diagnostic utility of salivary redox biomarkers in IR conditions. Biomarkers are biological indicators whose assessment allows qualitative or quantitative evaluation of pathological states and diseases. The biomarkers should differentiate patients from healthy controls (with high accuracy and specificity) and correlate with disease severity [23]. Of all the biomarkers we evaluated, salivary GSH and 4-HNE deserve special attention. Salivary GSH correlates negatively not only with body weight $(r=-0.667, p=0.035)$ and BMI $(r=-0.889, p=0.001)$, but also with plasma glucose $(r=-0.79, \quad p=0.006)$, insulin $(r=-0.783, \quad p=0.007)$, HOMA-IR $(r=-0.936, p=0.00007)$, and proinflammatory adipokines (leptin: $r=-0.757, p=0.011$; resistin: $r=0.685$, 


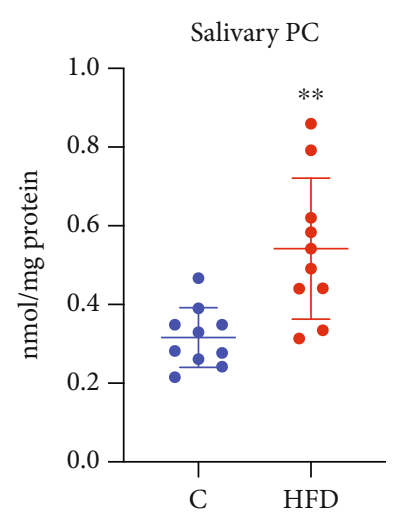

(a)

Saliva/plasma ratio PC

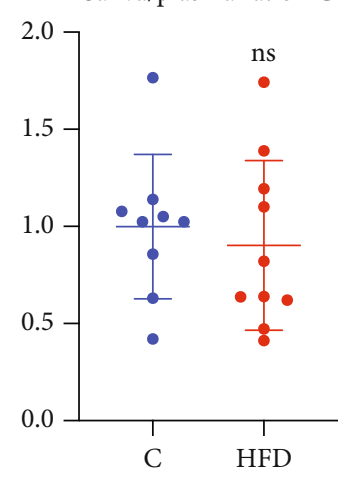

(c)

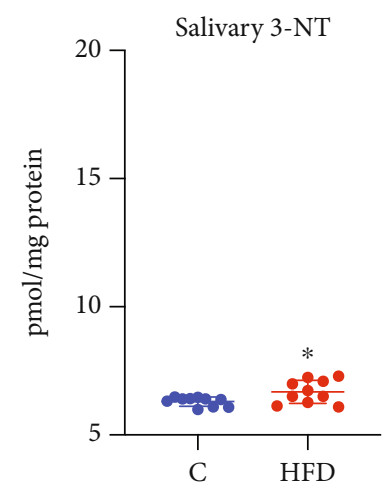

(e)

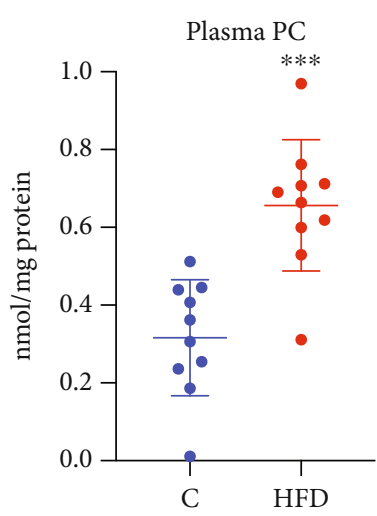

(b)

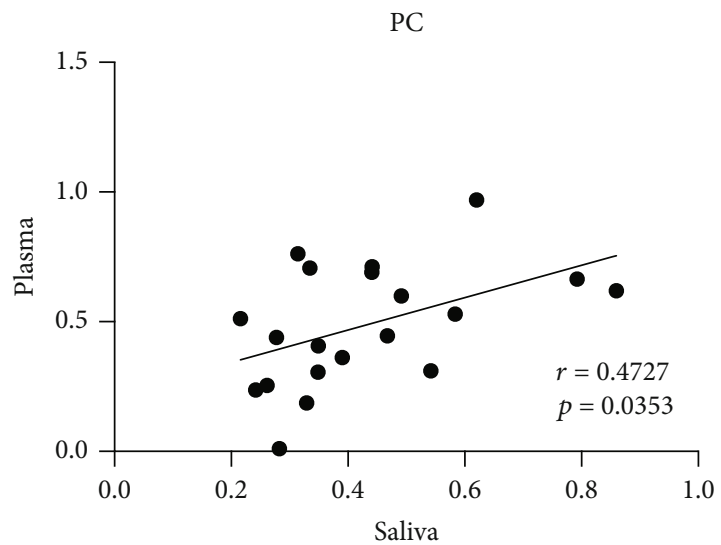

(d)

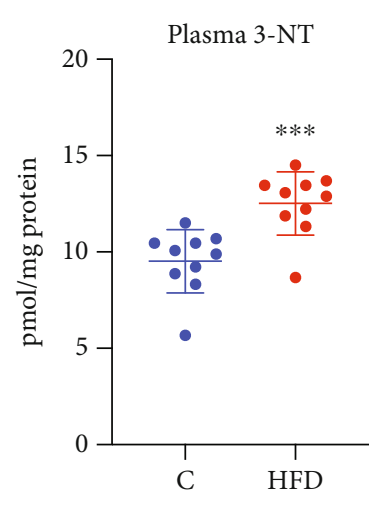

(f)

Figure 3: Continued. 

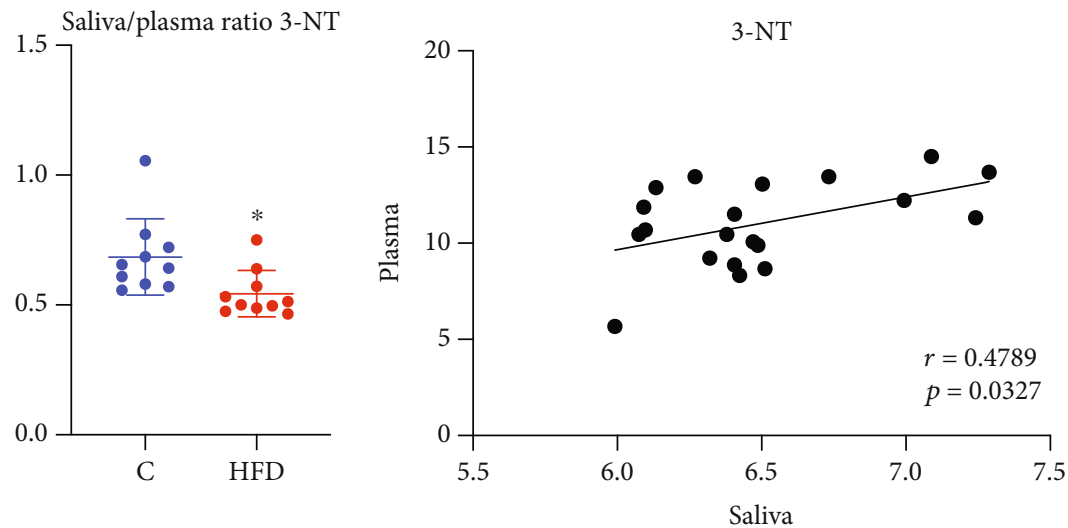

(g)

(h)
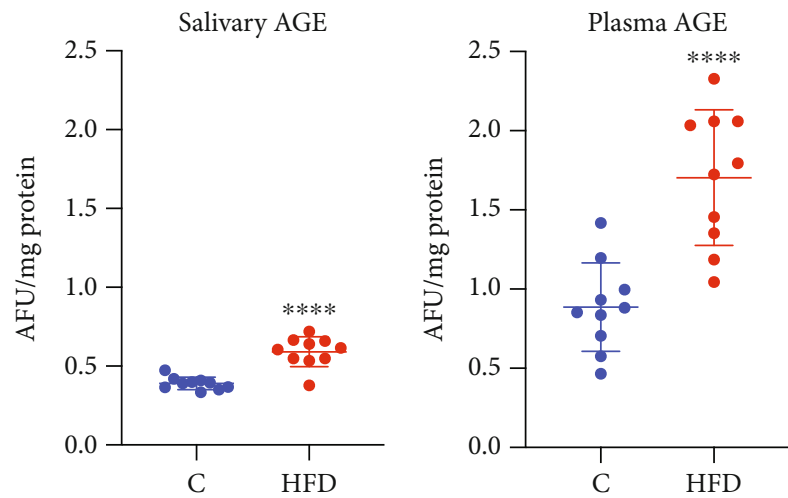

(i)
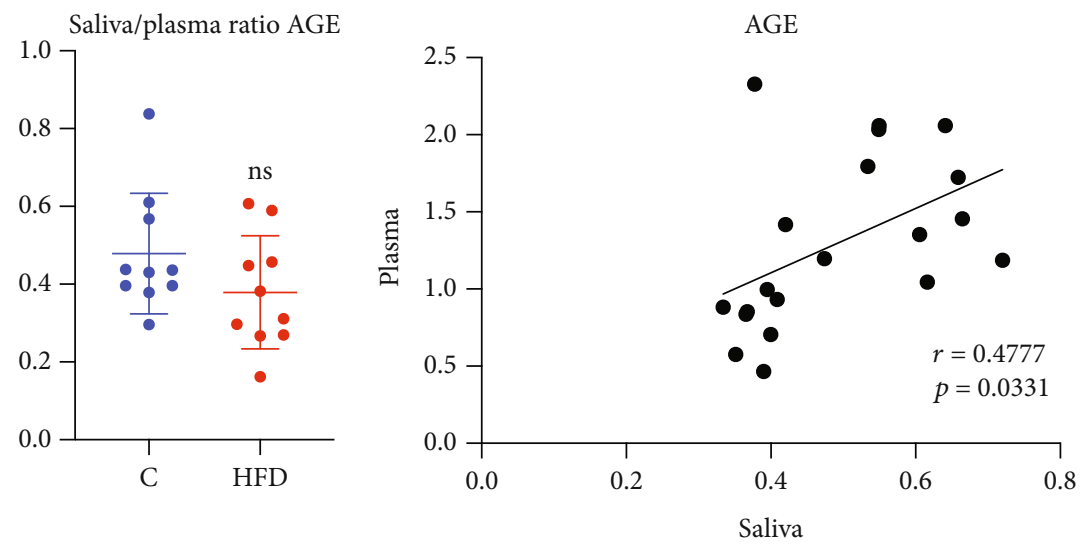

(k)

(1)

FIgUre 3: Continued. 


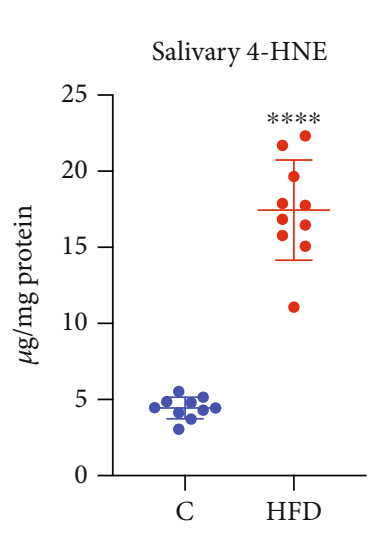

$(\mathrm{m})$

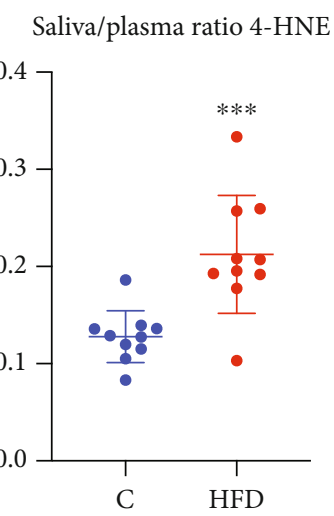

(o)

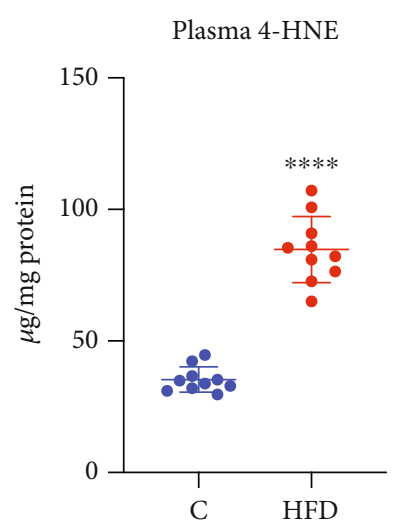

(n)

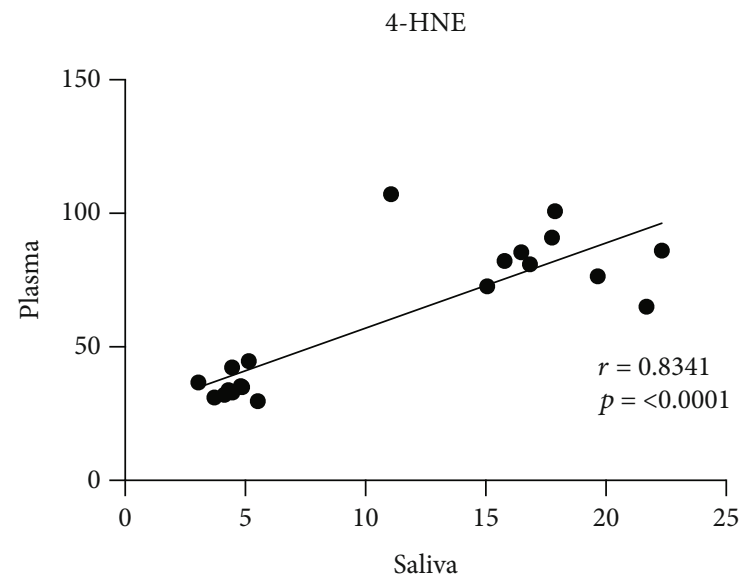

(p)

FIgURE 3: The effect of a high-fat diet (HFD) on salivary and plasma oxidative/nitrosative damage in rats. Abbreviations: C: control group; HFD: high-fat-diet group; 3-NT: 3-nitrotyrosine; 4-HNE: 4-hydroxynonenal; AGE: advanced glycation end products; PC: protein carbonyls; ${ }^{*} p<0.05$ vs. control; ${ }^{* *} p<0.01$ vs. control; ${ }^{* * *} p<0.001$ vs. control; ${ }^{* * * *} p<0.0001$ vs. control; ns: no significance.

$p=0.029 ;$ and TNF- $\alpha: r=-0.639, p=0.047)$. Multivariate regression analysis also showed that salivary GSH depends on the severity of obesity measured by BMI and reduced insulin sensitivity expressed as HOMA-IR. Therefore, GSH depletion may be associated with the progression of metabolic disturbances accompanying insulin resistance. Indeed, glutathione is one of the most critical intracellular antioxidants $[22,60]$. In addition to ROS scavenging and regenerating other antioxidants (e.g., vitamin E and GPx), GSH participates in restoring oxidatively modified proteins, lipids, and nucleic acids. It also acts as a major thiol buffer of the cell by regulating growth, differentiation, and apoptosis $[56,57]$. Reduced GSH level is a critical factor in increasing the intensity of membrane lipid peroxidation and ceramide accumulation in patients with obesity and IR [22, $61,62]$. In our study, this may be supported by the negative correlation between salivary GSH and 4-HNE $(r=0.663, p$ $=0.037)$. Indeed, lipids are particularly susceptible to oxidation. Lipid peroxidation products such as 4 -HNE cause further cellular damage, including disruption of gene expression/protein synthesis and uncoupling of oxidative phosphorylation [63]. 4-HNE can also increase inflammation by stimulating NADPH oxidase activity or activating macrophages [58, 64]. Therefore, the positive correlations of salivary 4-HNE and adipokines are not surprising (leptin: $r=0.626, p=0.053$; and resistin: $r=0.776, p=0.008$ ). However, salivary 4 -HNE correlates also with body weight $(r=0.605, p=0.044)$ and BMI $(r=0.854, p=0.002)$, as well as indicators of carbohydrate metabolism (glucose $(r=0.716, p=0.02)$; and HOMA-IR $(r=0.592, p=0.05))$. It is well known that IR is the most important cause of carbohydrate disorders. Hyperinsulinemia and insulin resistance are also independent factors in diabetes and cardiovascular disease $[4,5]$. Therefore, we used multiple regression to determine the diagnostic utility of salivary 4-HNE in the HFD-induced IR model. Regression analysis showed that this parameter highly depends on the HOMA-IR index and BMI. Its positive correlation with proinflammatory adipokines also demonstrates the diagnostic utility of salivary 4-HNE.

The oral cavity is a unique site in the body since it is exposed to many prooxidant factors such as air pollutants, diet, medications, dental materials, and other xenobiotics $[65,66]$. Although many antioxidants/oxidative stress products pass into saliva from the blood, their salivary content can not necessarily reflect the intensity of systemic oxidative 


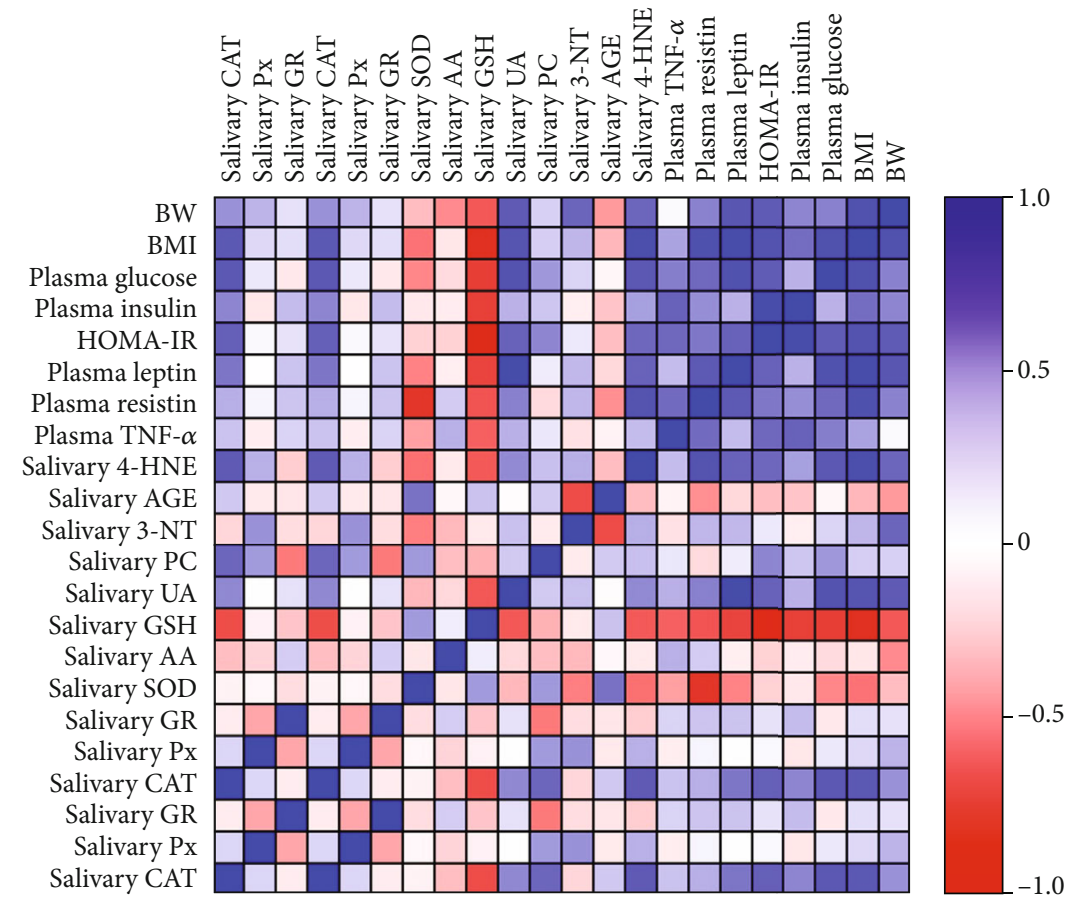

FIGURE 4: Correlations between salivary and plasma redox and metabolic parameters in rats. Abbreviations: 3-NT: 3-nitrotyrosine; 4-HNE: 4-hydroxynonenal; AA: ascorbic acid; AGE: advanced glycation end products; BMI: body mass index; BW: body weight; CAT: catalase; GSH: reduced glutathione; GR: glutathione reductase; HOMA-IR: homeostatic model assessment for insulin resistance; PC: protein carbonyls; Px: peroxidase; SOD: superoxide dismutase; TNF- $\alpha$ : tumor necrosis factor $\alpha$; UA: uric acid.

TABLE 2: Multiple regression analysis of the analyzed redox biomarkers.

\begin{tabular}{|c|c|c|c|c|c|c|}
\hline \multirow{2}{*}{ Parameter } & \multicolumn{3}{|c|}{$\beta 1:$ HOMA-IR } & \multicolumn{3}{|c|}{$\beta 2: \mathrm{BMI}$} \\
\hline & Estimate & $95 \% \mathrm{CI}$ & $p$ value & Estimate & $95 \% \mathrm{CI}$ & $p$ value \\
\hline $\begin{array}{l}\text { Salivary CAT } \\
\text { CA }\end{array}$ & 0.03866 & -0.1186 to 0.1959 & 0.5793 & 0.7877 & -0.6970 to 2.272 & 0.2499 \\
\hline Salivary Px & -4.2 & -23.23 to 14.83 & 0.6178 & 57.27 & -122.3 to 236.9 & 0.4754 \\
\hline Salivary GR & 0.07613 & -1.587 to 1.740 & 0.9169 & 1.356 & -14.35 to 17.06 & 0.844 \\
\hline Salivary SOD & 0.02902 & -0.02624 to 0.08427 & 0.2544 & -0.4925 & -1.014 to 0.02913 & 0.0607 \\
\hline Salivary AA & -1.039 & -5.541 to 3.462 & 0.602 & 3.56 & -38.93 to 46.05 & 0.8486 \\
\hline Salivary GSH & -0.2338 & -0.3665 to -0.1011 & 0.0042 & -1.44 & -2.693 to -0.1877 & 0.0298 \\
\hline Salivary UA & 0.01866 & -0.1543 to 0.1916 & 0.806 & 1.175 & -0.4573 to 2.808 & 0.1325 \\
\hline Salivary PC & 0.2107 & -0.1228 to 0.5443 & 0.1788 & -0.9228 & -4.071 to 2.226 & 0.5106 \\
\hline Salivary 3-NT & -0.2589 & -1.162 to 0.6447 & 0.5198 & 4.142 & -4.387 to 12.67 & 0.2885 \\
\hline Salivary AGE & -0.01719 & -0.2152 to 0.1808 & 0.8432 & -0.3393 & -2.209 to 1.530 & 0.6806 \\
\hline Salivary 4-HNE & -0.9857 & -4.660 to 2.688 & 0.546 & 47.96 & 13.29 to 82.64 & 0.0137 \\
\hline
\end{tabular}

Abbreviations: 3-NT: 3-nitrotyrosine; 4-HNE: 4-hydroxynonenal; AA: ascorbic acid; AGE: advanced glycation end products; BMI: body mass index; CAT: catalase; CI: confidence interval; GSH: reduced glutathione; GR: glutathione reductase; HOMA-IR: homeostatic model assessment for insulin resistance; PC: protein carbonyls; Px: peroxidase; SOD: superoxide dismutase; UA: uric acid.

stress. Indeed, in our study, salivary antioxidant enzymes did not correlate with serum activity (exception: SOD). Nevertheless, salivary nonenzymatic antioxidants reflect very well their plasma levels. We also observed positive correlations between the concentrations of protein and lipid oxidation products in plasma and saliva of IR rats. However, the highest correlation coefficients are found for GSH $(r=0.7993, p$ $<0.0001)$ and 4-HNE $(r=0.8341, p<0.0001)$. Therefore, salivary GSH and $4-\mathrm{HNE}$ can be used to assess systemic redox homeostasis. The results of the ROC analysis also demonstrated the high diagnostic utility of these biomarkers. ROC analysis evaluates the diagnostic power of the test and assesses the ability of the biomarker to discriminate between normal and abnormal values. Salivary GSH and 4-HNE differentiate with very high sensitivity (100\%) and specificity (100\%) between healthy animals and those with IR $(A U C=1.0)$. Thus, salivary GSH and 4-HNE meet all the criteria for a good laboratory biomarker [23]. Further 


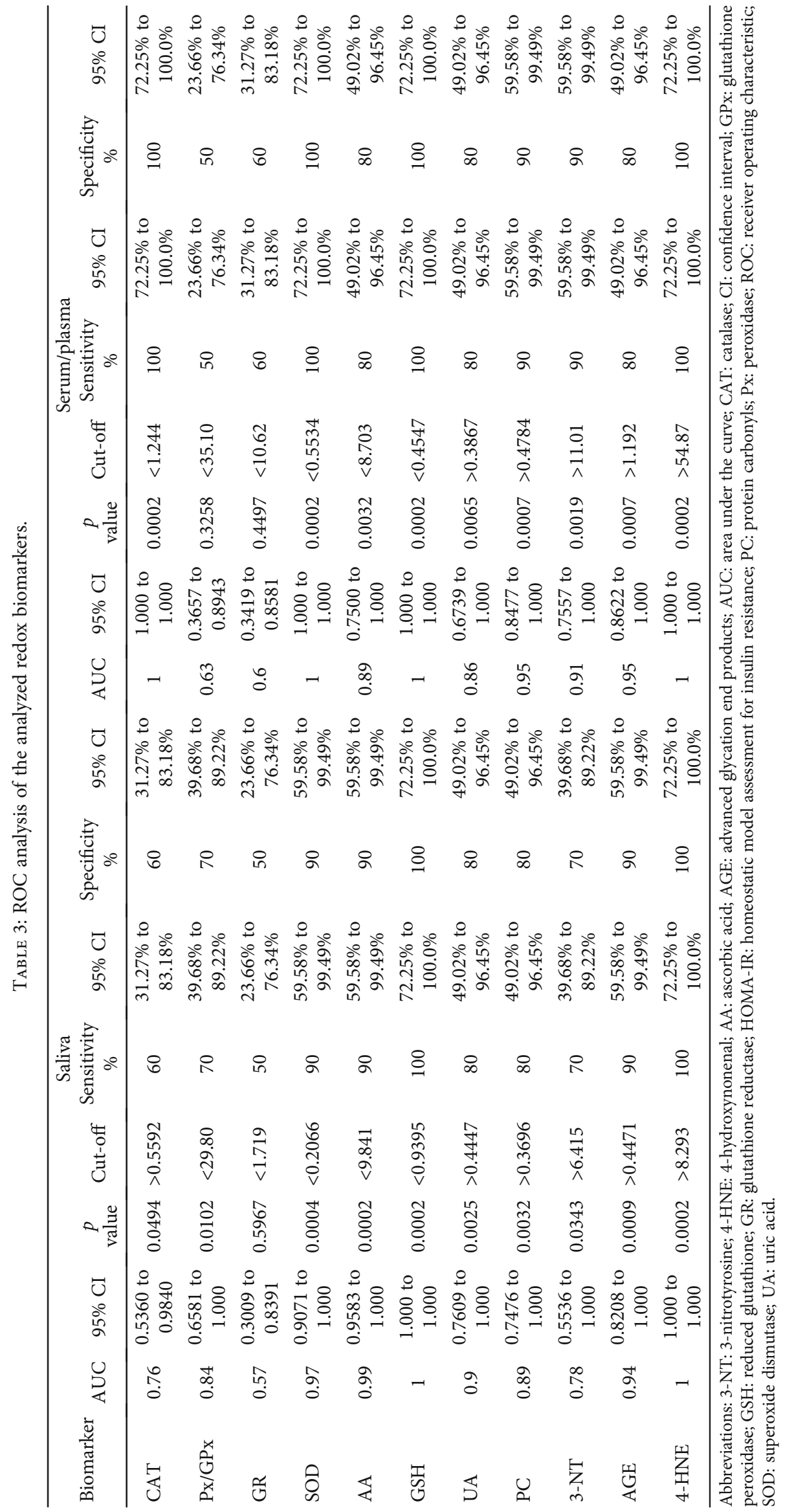


clinical studies are needed to evaluate their diagnostic potential. However, it should not be forgotten that in IR there is salivary hypofunction caused by disturbances in redox homeostasis $[12,67,68]$, which may affect the oxidative stress parameters in saliva. It is also necessary to compare salivary redox biomarkers in patients with IR and those with other metabolic, cardiovascular, and inflammatory diseases [44].

It is essential to note the limitations of our work. Because of the lack of Ethics Committee approval, we could not perform a hyperinsulinemic-euglycemic clamp. Additionally, we evaluated only the most commonly assessed redox biomarkers due to the low volume of saliva. Although the redox biomarkers described here distinguish IR rats and controls with high specificity and sensitivity, they may be nonspecific only for insulin resistance. Therefore, it is essential to evaluate salivary redox indicators also in other diseases with oxidative stress etiology. Nevertheless, this is the first study to show the potential use of saliva and oxidative stress biomarkers in monitoring IR progression.

\section{Conclusions}

(1) Saliva can be used to assess the systemic antioxidant status and the intensity of systemic oxidative stress

(2) Salivary GSH and 4-HNE may be potential biomarkers of IR progression

(3) There is a need for human clinical trials to evaluate the diagnostic utility of salivary redox biomarkers in IR conditions

\section{Materials and Methods}

5.1. Animals. The experiment was performed on male Wistar rats ( $R$. norvegicus; Wistar: cmd, outbred Cmdb:Wi) with an initial body weight of 50-60 g. The animals came from the Center for Experimental Medicine of the Medical University of Bialystok. The rats have been housed in individually ventilated laboratory cages at controlled temperatures $\left(20-22^{\circ} \mathrm{C}\right)$, under a standard condition of light from 6.00 a.m. to 6.00 p.m., and with free access to tap water and food.

The experimental procedures were approved by the institutional Committee for Ethics use of Animals in the University of Warmia and Mazury in Olsztyn, Poland (No. 21/2017).

After seven days of adaptation, the rats were divided into two groups of 10 individuals each.

(i) Group I-(C) control; rats receiving standard rodent diet (Research Diets, New Brunswick, NJ, USA, catalog number D12450J) containing $10 \%$ fat, $20 \%$ proteins, and $70 \%$ carbohydrates

(ii) Group II-(HFD) rats fed a high-fat diet (Research Diets, New Brunswick, NJ, USA catalog number D12492) containing $60 \%$ fat, $20 \%$ proteins, and $20 \%$ carbohydrates

Animals from control and HFD groups were fed the appropriate diet for eight weeks, while the body weight and food intake were monitored weekly. The body mass index
(BMI) was calculated using the formula BMI = body weight (g)/length ${ }^{2}\left(\mathrm{~cm}^{2}\right)$, and rat length was measured from the tip of the nose to the anus. BMI between 0.45 and $0.68 \mathrm{~g} / \mathrm{cm}^{2}$ was considered normal values, whereas BMI greater than $0.68 \mathrm{~g} / \mathrm{cm}^{2}$ indicated obesity $[69,70]$.

After eight weeks of the experiment, rats were fasted for $12 \mathrm{~h}$, anesthetized with sodium phenobarbital $(80 \mathrm{mg} / \mathrm{kg}$ body weight, intraperitoneally), and then the whole saliva was collected. The animals were peritoneally injected with $5 \mathrm{mg} / \mathrm{kg}$ BW pilocarpine nitrate (Sigma Chemical Co; St. Louis, MO, USA) in physiological saline. Five minutes later, a preweighted cotton ball was inserted into the oral cavity, and saliva was collected for five minutes [67, 68]. The volume of saliva was evaluated by subtracting the initial weight of cotton balls from their final weight. One $\mathrm{mg}$ of the collected saliva was considered to be one $\mu \mathrm{L}[67,68]$. Saliva was then centrifuged in Salivette tubes $\left(3000 \times \mathrm{g}, 4^{\circ} \mathrm{C}\right.$, $10 \mathrm{~min}$ ) to collect supernatant [71]. Next, whole blood was collected from the abdominal aorta into glass tubes (to obtain serum) and EDTA tubes (to obtain plasma) and centrifuged $\left(3000 \times \mathrm{g}, 4^{\circ} \mathrm{C}, 10 \mathrm{~min}\right)$. To protect against sample oxidation and proteolysis, the antioxidant butylated hydroxytoluene (BHT, ten $\mu \mathrm{L}$ of $0.5 \mathrm{M}$ BHT in acetonitrile per $1 \mathrm{~mL}$ sample; Sigma-Aldrich, Steinheim, Germany) and a protease inhibitor (Complete Mini Roche, France) were added to the collected saliva and plasma samples [72, 73]. All samples were stored at $-80^{\circ} \mathrm{C}$ but for no longer than six months.

Fasting blood glucose level was determined using the glucometer (Accu-Chek; Bayer, Germany). Fasting plasma insulin level was determined using a commercial ELISA kit according to the manufacturer's instructions (EIAab Science Inc. Wuhan; Wuhan, China). To confirm IR, the insulin sensitivity was determined using the homeostasis model assessment (HOMA $-\mathrm{IR})=$ fasting insulin $(\mathrm{U} / \mathrm{mL}) \times$ fasting glucose $(\mathrm{mM}) / 22.5$ [74]. According to the manufacturer's instructions, plasma adipocytokines (leptin, resistin, and TNF- $\alpha$ ) were determined using a commercial ELISA kit (EIAab Science Inc. Wuhan; Wuhan, China).

\subsection{Redox Assays}

5.2.1. Enzymatic Antioxidants. The activity of catalase (CAT) was analyzed spectrophotometrically by measuring the decomposition rate of hydrogen peroxide $\left(\mathrm{H}_{2} \mathrm{O}_{2}\right)$ in the sample at $240 \mathrm{~nm}$ wavelength [75]. One CAT unit was expressed as the amount of enzyme that decomposes $1 \mathrm{mmol}$ $\mathrm{H}_{2} \mathrm{O}_{2}$ within 1 minute. Salivary peroxidase ( $\left.\mathrm{Px}\right)$ activity was determined spectrophotometrically according to ManssonRahemtulla et al. method [76]. The absorbance changes in the reaction mixture containing 5,5' -dithiobis-2-nitrobenzoic acid (DTNB), potassium iodide (KI), and $\mathrm{H}_{2} \mathrm{O}_{2}$ were measured at $412 \mathrm{~nm}$ wavelength. The activity of glutathione peroxidase $(\mathrm{GPx})$ was assayed spectrophotometrically by the Paglia and Valentine method [77]. The absorbance was analyzed at $340 \mathrm{~nm}$, based on the conversion of reduced nicotinamide adenine dinucleotide phosphate (NADPH) to reduced nicotinamide adenine dinucleotide phosphate $(\mathrm{NADP}+)$. One unit of GPx activity was assumed to catalyze 
the oxidation of $1 \mathrm{mmol}$ of NADPH for 1 minute. The method of Mize and Langdon [78] was used to assess glutathione reductase (GR) activity. The absorbance of the samples was measured at $340 \mathrm{~nm}$ wavelength. One unit of GR activity was expressed as the amount of enzyme needed for the oxidation reaction of $1 \mu \mathrm{mol}$ of NADPH within $1 \mathrm{~min}$ ute. The activity of superoxide dismutase (SOD) was determined spectrophotometrically by measuring the absorbance changes accompanying adrenaline oxidation at $480 \mathrm{~nm}$ wavelength [79]. One unit of SOD activity was assumed to inhibit the oxidation of adrenaline by $50 \%$.

5.2.2. Nonenzymatic Antioxidants. The concentration of ascorbic acid (AA) was analyzed colorimetrically according to Jagota and Dani [80]. This method involves the reduction of the Folin phenol reagent under the influence of AA. The absorbance of the samples was measured at $760 \mathrm{~nm}$ wavelength. The content of reduced glutathione (GSH) was measured spectrophotometrically based on the reduction of DTNB to 2-nitro-5-mercaptobenzoic acid under the influence of GSH. The absorbance was measured at $412 \mathrm{~nm}$ wavelength [81]. The concentration of uric acid (UA) was analyzed colorimetrically by measuring the absorbance of 2,4,6-tripyridyl-s-triazine complex with iron ions and UA, using the commercial kit QuantiChromTM Uric Acid DIUA-250 (BioAssay Systems, Harward, CA, USA). The intensity of the examined sample was measured at $490 \mathrm{~nm}$ wavelength.

5.2.3. Oxidative/Nitrosative Damage. The concentration of protein carbonyl (PC) was determined colorimetrically based on the 2,4-dinitrophenylhydrazine (2,4-DNPH)'s reaction with carbonyl groups in the oxidatively damaged proteins. The intensity of the resultant hydrazone was measured at $355 \mathrm{~nm}$. PC content was calculated using an absorption coefficient for $2,4-\mathrm{DNPH}=22,000 \mathrm{M}^{-1} \mathrm{~cm}^{-1}$. According to the manufacturer's instructions, the concentration of 3-nitrotyrosine (3-NT) was measured by the ELISA method, using a commercial diagnostic kit (Immundiagnostik AG; Bensheim, Germany). Advanced glycation end product (AGE) level was analyzed spectrofluorimetrically by measuring the specific AGE fluorescence at $350 / 440 \mathrm{~nm}$ [82]. The samples were diluted in $0.02 \mathrm{M}$ PBS buffer for the AGE determination in plasma [44]. The concentration of 4-hydroxynonenal protein adduct (4-HNE) was determined by the ELISA method (OxiSelect ${ }^{\mathrm{TM}} \mathrm{HNE}$ Adduct Competitive ELISA Kit, Cell Biolabs Inc. San Diego, CA, USA), following the manufacturer's instructions provided in the package.

5.3. Statistical Analysis. Statistical analysis was performed using GraphPad Prism 8.4.3 for MacOS (GraphPad Software, La Jolla, USA). The Shapiro-Wilk test was used to determine the normality of distribution, while the Student's $t$-test was used to compare the IR group with the controls. The results were presented as mean \pm standard deviation (SD), and the value of $p<0.05$ was considered statistically significant. Pearson correlation coefficient was used to evaluate the relationships between redox biomarkers and meta- bolic parameters. To identify factors that determine the levels of redox biomarkers, we performed multiple regression analyses. HOMA-IR and BMI were included as independent variables; 95\% confidence intervals (CI) were reported along with regression parameters. Receiver operating characteristic (ROC) analysis was used to assess the diagnostic utility of the redox biomarkers. AUC (area under the curve) and optimal cut-off values were determined for each parameter that ensured high sensitivity with high specificity.

The number of animals was calculated a priori based on our previous preliminary study. Type I error $\alpha=0.05$ and statistical power (type II error) of 0.9 were considered. Statistical test assumptions were validated for all the analyses performed. The minimum number of rats in one group was seven, and therefore, the analysis was performed on ten individuals.

\section{Data Availability}

The datasets generated for this study are available on request to the corresponding author.

\section{Ethical Approval}

The study was approved by the institutional Committee for Ethics use of Animals in the University of Warmia and Mazury in Olsztyn, Poland (No. 21/2017).

\section{Conflicts of Interest}

The authors declare no conflict of interest.

\section{Authors' Contributions}

Conceptualization was done by M.M. and A.Z.; data curation was done by M.M. and A.Z.; formal analysis was done by M.M.; funding acquisition was done by M.M. and A.Z.; investigation was done by M.M.; methodology was done by M.M. and C.P.; material collection was done by M.M. and M.Z.P.; supervision was done by J.R.L. and A.Z.; validation was done by M.M.; visualization was done by M.M.; writing-original draft was done by M.M.; writing-review and editing was done by M.M. and A.Z. All authors have read and agreed to the published version of the manuscript.

\section{Acknowledgments}

This work was granted by the Medical University of Bialystok, Poland (grant numbers: SUB/1/DN/21/002/3330 and SUB/1/DN/21/002/1209). Dr. Mateusz Maciejczyk was supported by the Foundation for Polish Science (FNP).

\section{References}

[1] M. A. B. Khan, M. J. Hashim, J. K. King, R. D. Govender, H. Mustafa, and J. Al Kaabi, "Epidemiology of type 2 diabetes-global burden of disease and forecasted trends," Journal of Epidemiology and Global Health, vol. 10, 2019.

[2] X. Lin, Y. Xu, X. Pan et al., "Global, regional, and national burden and trend of diabetes in 195 countries and territories: an 
analysis from 1990 to 2025," Scientific Reports, vol. 10, no. 1, p. 14790, 2020.

[3] S. E. Kahn, M. E. Cooper, and S. Del Prato, "Pathophysiology and treatment of type 2 diabetes: perspectives on the past, present, and future," Lancet, vol. 383, no. 9922, pp. 10681083, 2014.

[4] D. Yazıc1 and H. Sezer, "Insulin resistance, obesity and lipotoxicity," In Advances in Experimental Medicine and Biology, vol. 960, pp. 277-304, 2017.

[5] X. Tian, C. Yan, M. Liu et al., "CREG1 heterozygous mice are susceptible to high fat diet-induced Obesity and insulin resistance," PLoS One, vol. 12, no. 5, p. e0176873, 2017.

[6] G. Solinas and M. Karin, "JNK1 and IKK $\beta$ : molecular links between obesity and metabolic dysfunction," The FASEB Journal, vol. 24, no. 8, pp. 2596-2611, 2010.

[7] S. Tangvarasittichai, "Oxidative stress, insulin resistance, dyslipidemia and type 2 diabetes mellitus," World Journal of Diabetes, vol. 6, no. 3, pp. 456-480, 2015.

[8] J. Styskal, H. Van Remmen, A. Richardson, and A. B. Salmon, "Oxidative stress and diabetes: what can we learn about insulin resistance from antioxidant mutant mouse models?," Free Radical Biology \& Medicine, vol. 52, no. 1, pp. 46-58, 2012.

[9] J. L. Rains and S. K. Jain, "Oxidative stress, insulin signaling, and diabetes," Free Radical Biology \& Medicine, vol. 50, no. 5, pp. 567-575, 2011.

[10] V. Aguirre, T. Uchida, L. Yenush, R. Davis, and M. F. White, "The c-Jun $\mathrm{NH}_{2}$-terminal Kinase Promotes Insulin Resistance during Association with Insulin Receptor Substrate-1 and Phosphorylation of Ser," The Journal of Biological Chemistry, vol. 275, no. 12, pp. 9047-9054, 2000.

[11] G. Verdile, K. N. Keane, V. F. Cruzat et al., "Inflammation and oxidative stress: the molecular connectivity between insulin resistance, obesity, and Alzheimer's disease," Mediators of Inflammation, vol. 2015, 17 pages, 2015.

[12] A. Zalewska, M. Maciejczyk, J. Szulimowska, M. Imierska, and A. Błachnio-Zabielska, "High-fat diet affects ceramide content, disturbs mitochondrial redox balance, and induces apoptosis in the submandibular glands of mice," Biomolecules, vol. 9, no. 12, p. 877, 2019.

[13] G. Poli, G. Leonarduzzi, F. Biasi, and E. Chiarpotto, "Oxidative stress and cell signalling," Current Medicinal Chemistry, vol. 11, no. 9, 2004.

[14] N. Andrieu-Abadie, V. Gouazé, R. Salvayre, and T. Levade, "Ceramide in apoptosis signaling: relationship with oxidative stress," Free Radical Biology \& Medicine, vol. 31, no. 6, pp. 717-728, 2001.

[15] F. Giacco and M. Brownlee, "Oxidative stress and diabetic complications," Circulation Research, vol. 107, no. 9, pp. 1058-1070, 2010.

[16] B. Choromańska, P. Myśliwiec, J. Dadan, A. Maleckas, A. Zalewska, and M. Maciejczyk, "Effects of age and gender on the redox homeostasis of morbidly obese people," Free Radical Biology and Medicine, 2021.

[17] P. Codoñer-Franch, S. Tavárez-Alonso, R. Murria-Estal, M. Tortajada-Girbés, R. Simó-Jordá, and E. Alonso-Iglesias, "Elevated advanced oxidation protein products (AOPPs) indicate metabolic risk in severely obese children," Nutrition, Metabolism, and Cardiovascular Diseases, vol. 22, no. 3, pp. 237-243, 2012.

[18] G. L. Rodríguez-González, L. A. Reyes-Castro, C. J. Bautista et al., "Maternal obesity accelerates rat offspring metabolic ageing in a sex-dependent manner," The Journal of Physiology, vol. 597, no. 23, pp. 5549-5563, 2019.

[19] K. Park, M. Gross, D.-H. Lee et al., "Oxidative stress and insulin resistance: the coronary artery risk development in young adults study," Diabetes Care, vol. 32, no. 7, pp. 1302-1307, 2009.

[20] A. Khosrowbeygi, M. Gholami, P. Zarei, B. Sadeghi Sedeh, M. Reza Rezvanfar, and M. Reza Rezvanfar, "Correlations between biomarkers of oxidative stress, glycemic control and insulin resistance in women with type 2 diabetes," Clinical Diabetology, vol. 8, pp. 277-283, 2020.

[21] S. Paramasivan, S. S. Adav, S. C. Ngan et al., "Serum albumin cysteine trioxidation is a potential oxidative stress biomarker of type 2 diabetes mellitus," Scientific Reports, vol. 10, no. 1, p. $6475,2020$.

[22] B. Choromańska, P. Myśliwiec, M. Łuba et al., "The impact of hypertension and metabolic syndrome on nitrosative stress and glutathione metabolism in patients with morbid obesity," Oxidative Medicine and Cellular Longevity, vol. 2020, 10 pages, 2020.

[23] R. M. Califf, "Biomarker definitions and their applications," Experimental Biology and Medicine (Maywood, N.J.), vol. 243, no. 3, pp. 213-221, 2018.

[24] M. Maciejczyk, M. Bielas, A. Zalewska, and K. Gerreth, "Salivary biomarkers of oxidative stress and inflammation in stroke patients: from basic research to clinical practice," Oxidative Medicine and Cellular Longevity, vol. 2021, Article ID 5545330, 2021.

[25] J. M. Yoshizawa, C. A. Schafer, J. J. Schafer, J. J. Farrell, B. J. Paster, and D. T. W. Wong, "Salivary biomarkers: toward future clinical and diagnostic utilities," Clinical Microbiology Reviews, vol. 26, no. 4, pp. 781-791, 2013.

[26] M. A. Javaid, A. S. Ahmed, R. Durand, and S. D. Tran, Saliva as a Diagnostic Tool for Oral and Systemic Diseases, J. Oral Biol, Craniofacial Res, 2016.

[27] N. Malathi, S. Mythili, and H. R. Vasanthi, "Salivary diagnostics: a brief review," ISRN Dentistry, vol. 2014, 8 pages, 2014.

[28] M. Battino, M. S. Ferreiro, I. Gallardo, H. N. Newman, and P. Bullon, "The antioxidant capacity of saliva," Journal of Clinical Periodontology, vol. 29, no. 3, pp. 189-194, 2002.

[29] M. Maciejczyk, A. Zalewska, and K. Gerreth, "Salivary redox biomarkers in selected neurodegenerative diseases," Journal of Clinical Medicine, vol. 9, no. 2, p. 497, 2020.

[30] L. Malathi, E. Rajesh, N. Aravindha Babu, and S. Jimson, "Saliva as a diagnostic tool," Biomedical and Pharmacology Journal, vol. 9, no. 2, pp. 867-870, 2016.

[31] S. E. Park, C.-Y. Park, and G. Sweeney, "Biomarkers of insulin sensitivity and insulin resistance: past, present and future," Critical Reviews in Clinical Laboratory Sciences, vol. 52, no. 4, pp. 180-190, 2015.

[32] Y.-H. Lee and D. T. Wong, "Saliva: an emerging biofluid for early detection of diseases," American Journal of Dentistry, vol. 22, no. 4, pp. 241-248, 2009.

[33] J. Pasic and J. C. Pickup, "Salivary insulin in normal and type I diabetic subjects," Diabetes Care, vol. 11, pp. 489-494, 1988.

[34] M.-L. Hartman, J. M. Goodson, P. Shi et al., "Unhealthy phenotype as indicated by salivary biomarkers: glucose, insulin, VEGF-A, and IL-12p70 in obese Kuwaiti adolescents," Journal of Obesity, vol. 2016, Article ID 6860240, 2016.

[35] R. K. D. Ephraim, E. O. Anto, E. Acheampong et al., "Fasting salivary glucose levels is not a better measure for identifying 
diabetes mellitus than serum or capillary blood glucose levels: comparison in a Ghanaian population," Heliyon, vol. 5, article e01286, 2019.

[36] M. Maciejczyk, K. Taranta-Janusz, A. Wasilewska, A. Kossakowska, and A. Zalewska, "A case-control study of salivary redox homeostasis in hypertensive children. Can salivary uric acid be a marker of hypertension?," Journal of Clinical Medicine, vol. 9, 2020.

[37] B. N. M. A. R. Diajil, Oxidative Stress Status in Hypertensive Patients on Capoten Treatment, Int. J. Sci, 2018.

[38] A. Zalewska, A. Kossakowska, K. Taranta-Janusz et al., "Dysfunction of salivary glands, disturbances in salivary antioxidants and increased oxidative damage in saliva of overweight and obese adolescents," Journal of Clinical Medicine, vol. 9, no. 2, p. $548,2020$.

[39] K. Fejfer, P. Buczko, M. Niczyporuk et al., "Oxidative modification of biomolecules in the nonstimulated and stimulated saliva of patients with morbid obesity treated with bariatric surgery," BioMed Research International, vol. 2017, 8 pages, 2017.

[40] E. O. Chielle and J. N. Casarin, "Evaluation of salivary oxidative parameters in overweight and obese young adults," Archives of Endocrinology and Metabolism, vol. 61, no. 2, pp. 152-159, 2017.

[41] G. Bibi, Y. Green, and R. M. Nagler, "Compositional and oxidative analysis in the saliva and serum of predialysis chronic kidney disease patients and end-stage renal failure patients on peritoneal dialysis," Therapeutic Apheresis and Dialysis, vol. 12, no. 2, pp. 164-170, 2008.

[42] M. Maciejczyk, J. Szulimowska, K. Taranta-Janusz, A. Wasilewska, and A. Zalewska, "Salivary gland dysfunction, protein glycooxidation and nitrosative stress in children with chronic kidney disease," Journal of Clinical Medicine, vol. 9, no. 5, p. 1285, 2020.

[43] S. Ghimenti, T. Lomonaco, F. G. Bellagambi et al., "Salivary lactate and 8-isoprostaglandin $\mathrm{F}_{2 \alpha}$ as potential non-invasive biomarkers for monitoring heart failure: a pilot study," Scientific Reports, vol. 10, no. 1, p. 7441, 2020.

[44] A. Klimiuk, A. Zalewska, R. Sawicki, M. Knapp, and M. Maciejczyk, "Salivary oxidative stress increases with the progression of chronic heart failure," Journal of Clinical Medicine, vol. 9, no. 3, p. 769, 2020.

[45] K. Morawska, M. Maciejczyk, Ł. Popławski, A. PopławskaKita, A. Krętowski, and A. Zalewska, "Enhanced salivary and general oxidative stress in Hashimoto's thyroiditis women in euthyreosis," Journal of Clinical Medicine, vol. 9, no. 7, p. 2102, 2020.

[46] M. Atar, A. Tan, Z. Ay, and O. Pirgon, Investigation of oxidative effect in saliva and gingival groove fluids in children with Hashimoto thyroiditis, Horm. Res. Paediatr, 2019.

[47] A. Klimiuk, M. Maciejczyk, M. Choromańska, K. Fejfer, N. Waszkiewicz, and A. Zalewska, "Salivary redox biomarkers in different stages of dementia severity," Journal of Clinical Medicine, vol. 8, no. 6, p. 840, 2019.

[48] J. M. Galindez, L. Juwara, M. Cressatti, M. Gornitsky, A. M. Velly, and H. M. Schipper, "Salivary heme oxygenase-1: a potential biomarker for central neurodegeneration," Journal of Central Nervous System Disease, vol. 13, 2021.

[49] E. Sánchez, J. A. Baena-Fustegueras, M. C. de la Fuente et al., "Advanced glycation end-products in morbid obesity and after bariatric surgery: when glycemic memory starts to fail," Endocrinología, Diabetes y Nutrición, vol. 64, no. 1, pp. 4-10, 2017.
[50] M. Gornitsky, A. M. Velly, S. Mohit et al., "Altered levels of salivary 8-oxo-7-hydrodeoxyguanosine in breast cancer," JDR Clinical \& Translational Research, vol. 1, no. 2, pp. 171-177, 2016.

[51] J. Szendroedi, E. Phielix, and M. Roden, "The role of mitochondria in insulin resistance and type 2 diabetes mellitus," Nature Reviews. Endocrinology, vol. 8, no. 2, pp. 92-103, 2012.

[52] M. K. Montgomery and N. Turner, "Mitochondrial dysfunction and insulin resistance: an update," Endocrine Connections, vol. 4, 2015.

[53] N. Esser, S. Legrand-Poels, J. Piette, A. J. Scheen, and N. Paquot, "Inflammation as a link between obesity, metabolic syndrome and type 2 diabetes," Diabetes Research and Clinical Practice, vol. 105, no. 2, pp. 141-150, 2014.

[54] R. Barazzoni, G. Gortan Cappellari, M. Ragni, and E. Nisoli, "Insulin resistance in obesity: an overview of fundamental alterations," Eating and Weight Disorders, vol. 23, no. 2, pp. 149-157, 2018.

[55] S. Furukawa, T. Fujita, M. Shimabukuro et al., "Increased oxidative stress in obesity and its impact on metabolic syndrome," The Journal of Clinical Investigation, vol. 114, no. 12, pp. 1752 1761, 2004.

[56] K. Aquilano, S. Baldelli, and M. R. Ciriolo, "Glutathione: new roles in redox signaling for an old antioxidant," Frontiers in Pharmacology, vol. 5, 2014.

[57] A. Scirè, L. Cianfruglia, C. Minnelli et al., "Glutathione compartmentalization and its role in glutathionylation and other regulatory processes of cellular pathways," BioFactors, vol. 45, no. 2, pp. 152-168, 2019.

[58] A. Ayala, M. F. Muñoz, and S. Argüelles, "Lipid peroxidation: production, metabolism, and signaling mechanisms of malondialdehyde and 4-hydroxy-2-nonenal," Oxidative Medicine and Cellular Longevity, vol. 2014, 31 pages, 2014.

[59] S. Pizzimenti, C. Toaldo, P. Pettazzoni, M. U. Dianzani, and G. Barrera, "The "two-faced" effects of reactive oxygen species and the lipid peroxidation product 4-hydroxynonenal in the hallmarks of cancer," Cancers (Basel)., vol. 2, no. 2, pp. 338$363,2010$.

[60] J. Langhardt, G. Flehmig, N. Klöting et al., "Effects of weight loss on glutathione peroxidase 3 serum concentrations and adipose tissue expression in human obesity," Obesity Facts, vol. 11, no. 6, pp. 475-490, 2018.

[61] E. E. Bankoglu, F. Seyfried, C. Arnold et al., "Reduction of DNA damage in peripheral lymphocytes of obese patients after bariatric surgery-mediated weight loss," Mutagenesis, vol. 33, no. 1, pp. 61-67, 2018.

[62] D. Nguyen, S. L. Samson, V. T. Reddy, E. V. Gonzalez, and R. V. Sekhar, "Impaired mitochondrial fatty acid oxidation and insulin resistance in aging: novel protective role of glutathione," Aging Cell, vol. 12, no. 3, pp. 415-425, 2013.

[63] M. A. Elrayess, S. Almuraikhy, W. Kafienah et al., "4-hydroxynonenal causes impairment of human subcutaneous adipogenesis and induction of adipocyte insulin resistance," Free Radical Biology \& Medicine, vol. 104, pp. 129-137, 2017.

[64] M. P. Mattson, "Roles of the lipid peroxidation product 4hydroxynonenal in obesity, the metabolic syndrome, and associated vascular and neurodegenerative disorders," Experimental Gerontology, vol. 44, no. 10, pp. 625-633, 2009.

[65] I. Zieniewska, M. Maciejczyk, and A. Zalewska, "The effect of selected dental materials used in conservative dentistry, endodontics, surgery, and orthodontics as well as during the 
periodontal treatment on the redox balance in the oral cavity," International Journal of Molecular Sciences, vol. 21, no. 24, p. 9684, 2020.

[66] K. Avezov, A. Z. Reznick, and D. Aizenbud, "Oxidative stress in the oral cavity: sources and pathological outcomes," Respiratory Physiology \& Neurobiology, vol. 209, pp. 91-94, 2015.

[67] A. Zalewska, I. Szarmach, M. Żendzian-Piotrowska, and M. Maciejczyk, "The effect of N-acetylcysteine on respiratory enzymes, ADP/ATP ratio, glutathione metabolism, and nitrosative stress in the salivary gland mitochondria of insulin resistant rats," Nutrients, vol. 12, no. 2, p. 458, 2020.

[68] U. Kołodziej, M. Maciejczyk, A. Miasko et al., "Oxidative modification in the salivary glands of high fat-diet induced insulin resistant rats," Frontiers in Physiology, vol. 8, 2017.

[69] E. L. B. Novelli, Y. S. Diniz, C. M. Galhardi et al., "Anthropometrical parameters and markers of obesity in rats," Laboratory Animals, vol. 41, no. 1, pp. 111-119, 2007.

[70] M. J. T. Engelbregt, M. M. van Weissenbruch, C. Popp-Snijders, P. Lips, and H. A. Delemarre-van de Waal, "Body mass index, body composition, and leptin at onset of puberty in male and female rats after intrauterine growth retardation and after early postnatal food restriction," Pediatric Research, vol. 50, pp. 474-478, 2001.

[71] A. Skutnik-Radziszewska, M. Maciejczyk, I. Flisiak et al., "Enhanced inflammation and nitrosative stress in the saliva and plasma of patients with plaque psoriasis," Journal of Clinical Medicine, vol. 9, no. 3, p. 745, 2020.

[72] A. Zalewska, S. Zięba, P. Kostecka-Sochoń et al., "NAC supplementation of hyperglycemic rats prevents the development of insulin resistance and improves antioxidant status but only alleviates general and salivary gland oxidative stress," Oxidative Medicine and Cellular Longevity, vol. 2020, 15 pages, 2020.

[73] M. Maciejczyk, J. Matczuk, M. Żendzian-Piotrowska et al., "Eight-week consumption of high-sucrose diet has a prooxidant effect and alters the function of the salivary glands of rats," Nutrients, vol. 10, no. 10, p. 1530, 2018.

[74] J. Cacho, J. Sevillano, J. de Castro, E. Herrera, and M. P. Ramos, "Validation of simple indexes to assess insulin sensitivity during pregnancy in Wistar and Sprague-Dawley rats," American Journal of Physiology-Endocrinology and Metabolism, vol. 295, pp. E1269-E1276, 2008.

[75] H. Aebi, "13 Catalase in vitro," Methods in Enzymology, vol. 105, pp. 121-126, 1984.

[76] B. Mansson-Rahemtulla, D. C. Baldone, K. M. Pruitt, and F. Rahemtulla, "Specific assays for peroxidases in human saliva," Archives of Oral Biology, vol. 31, pp. 661-668, 1986.

[77] D. E. Paglia and W. N. Valentine, "Studies on the quantitative and qualitative characterization of erythrocyte glutathione peroxidase," The Journal of Laboratory and Clinical Medicine, vol. 70, pp. 158-169, 1967.

[78] C. E. MIZE and R. G. LANGDON, "Hepatic glutathione reductase: I. Purification and general kinetic properties," The Journal of Biological Chemistry, vol. 237, pp. 1589-1595, 1962.

[79] H. P. Misra and I. Fridovich, "The role of superoxide anion in the autoxidation of epinephrine and a simple assay for superoxide dismutase," The Journal of Biological Chemistry, vol. 247 , pp. $3170-3175,1972$.
[80] S. K. Jagota and H. M. Dani, "A new colorimetric technique for the estimation of vitamin C using Folin phenol reagent," Analytical Biochemistry, vol. 127, no. 1, pp. 178-182, 1982.

[81] G. L. Ellman, “Tissue sulfhydryl groups,” Archives of Biochemistry and Biophysics, vol. 82, no. 1, pp. 70-77, 1959.

[82] M. Kalousová, T. Zima, V. Tesař, and J. Lachmanová, "Advanced glycation end products and advanced oxidation protein products in hemodialyzed patients," Blood Purification, vol. 20, no. 6, pp. 531-536, 2002. 